\title{
Metallicity and the spectral energy distribution and spectral types of dwarf O-stars
}

\author{
M. R. Mokiem ${ }^{1}$, N. L. Martín-Hernández ${ }^{2, \star}$, A. Lenorzer ${ }^{1}$, A. de Koter ${ }^{1}$, and A. G. G. M. Tielens ${ }^{2,3}$ \\ 1 Astronomical Institute Anton Pannekoek, University of Amsterdam, Kruislaan 403, 1098 SJ Amsterdam, The Netherlands \\ 2 Kapteyn Institute, PO Box 800, 9700 AV Groningen, The Netherlands \\ 3 SRON, National Institute for Space Reasearch, PO Box 800, 9700 AV Groningen, The Netherlands
}

Received 18 November 2002 / Accepted 11 February 2004

\begin{abstract}
We present a systematic study of the effect of metallicity on the stellar spectral energy distribution (SED) of O main sequence (dwarf) stars, focussing on the hydrogen and helium ionizing continua, and on the optical and near-IR lines used for spectral classification. The spectra are based on non-LTE line blanketed atmosphere models with stellar winds calculated using the CMFGEN code of Hillier \& Miller (1998). We draw the following conclusions. First, we find that the total number of Lyman photons emitted is almost independent of line blanketing effects and metallicity for a given effective temperature. This is because the flux that is blocked by the forest of metal lines at $\lambda<600 \AA$ is redistributed mainly within the Lyman continuum. Second, the spectral type, as defined by the ratio of the equivalent widths of He I $\lambda 4471$ and He II $\lambda 4542$, is shown to depend noticeably on the microturbulent velocity in the atmosphere, on metallicity and, within the luminosity class of dwarfs, on gravity. Third, we confirm the decrease in $T_{\text {eff }}$ for a given spectral type due to the inclusion of line blanketing recently found by e.g. Martins et al. (2002). Finally, we find that the SED below $\sim 450 \AA$ is highly dependent on metallicity. This is reflected in the behaviour of nebular fine-structure line ratios such as [Ne III]/[Ne II] 15.5/12.8 and [Ar III]/[Ar II] 9.0/7.0 $\mu \mathrm{m}$. This dependence complicates the use of these nebular ratios as diagnostic tools for the effective temperature determination of the ionizing stars in $\mathrm{H}$ II regions and for age dating of starburst regions in galaxies.
\end{abstract}

Key words. stars: atmospheres - stars: early-type - stars: fundamental parameters - stars: abundances - ISM: HII regions ISM: planetary nebulae: general

\section{Introduction}

Massive stars are a dominant force in the evolution of the interstellar medium of galaxies. The extreme ultraviolet photons of massive stars can ionize surrounding atomic hydrogen gas. This ionized gas is heated by the photo electrons and cooled through forbidden line radiation of trace species such as oxygen, resulting in a temperature of some $10^{4} \mathrm{~K}$. Besides this energetic coupling through the radiation field, massive stars can also influence their surroundings dynamically. The high pressure of the ionized gas will drive shock waves in their surroundings, sweeping up the ambient molecular gas. Moreover, these stars also have strong stellar winds and explode as type II supernovae, both of which provide kinetic energy to the interstellar gas. Understanding the characteristics of massive stars and their interaction with their environment is therefore a key problem in astrophysics.

The stellar spectral energy distribution in the extreme ultraviolet (EUV) controls the ionization structure of

\footnotetext{
Send offprint requests to: M. R. Mokiem,

e-mail: mokiem@science.uva.nl

* Present address: Geneva Observatory, 1290 Sauverny, Switzerland.
}

H II regions. However, because of the high opacity of neutral gas in the EUV, this wavelength range cannot be observed directly. Spectral typing of stars is generally done through optical (e.g. Conti \& Alschuler 1971; Conti \& Frost 1977; Mathys 1988) or near-IR features (e.g. Hanson et al. 1996; Meyer et al. 1998; Hanson et al. 1998; Lenorzer et al. 2002). Alternatively, observed nebular ionization structures can be used to probe the ionizing fluxes of the stars energizing the medium. This technique is now widely used to determine the properties of stars in the EUV and has even evolved to a more general astronomical tool, particularly for the study of regions where individual stars cannot be directly typed (e.g. Oey et al. 2000; Takahashi et al. 2000; Okamoto et al. 2001; Morisset et al. 2002). Moreover, because the hardness of the EUV radiation field is a good measure of the spectral type of the ionizing star and because later spectral types live longer, the observed ionization structure can be used as an age indicator of a starburst region (e.g. Crowther et al. 1999; Thornley et al. 2000; Spoon et al. 2000).

In recent years, some problems with the latter technique have emerged. For example, for the well-studied H II region G29.96-0.02, the spectral type derived from direct observations of the stellar lines in the near-IR (Watson \& Hanson 1997; Martín-Hernández et al. 2003) indicates a much earlier spectral 
Table 1. Stellar and wind parameters of the model grid stars at solar metallicity.

\begin{tabular}{cccccccccccc}
\hline \hline Model & $\begin{array}{c}T_{\text {eff }} \\
(\mathrm{K})\end{array}$ & $\frac{M_{\star}}{M_{\odot}}$ & $\frac{R_{\star}}{R_{\odot}}$ & $\begin{array}{c}\log g \\
\left(\mathrm{~cm} \mathrm{~s}^{-2}\right)\end{array}$ & $\log \frac{L_{\star}}{L_{\odot}}$ & $\begin{array}{c}H_{\star} \\
\left(10^{-4} R_{\star}\right)\end{array}$ & $\begin{array}{c}\log \dot{M} \\
\left(M_{\odot} / \mathrm{yr}\right)\end{array}$ & $\beta$ & $\begin{array}{c}V_{\infty} \\
\left(\mathrm{km} \mathrm{s}^{-1}\right)\end{array}$ & $\begin{array}{c}\log Q_{0} \\
\left(\mathrm{photons} \mathrm{s}^{-1}\right)\end{array}$ \\
\hline 1 & 51230 & 87.6 & 13.2 & 4.139 & 6.032 & 7.703 & -5.209 & 0.8 & 3450 & 49.87 & 49.38 \\
2 & 48670 & 68.9 & 12.3 & 4.096 & 5.882 & 8.308 & -5.375 & 0.8 & 3240 & 49.69 & 49.18 \\
3 & 46120 & 56.6 & 11.4 & 4.077 & 5.772 & 8.375 & -5.599 & 0.8 & 3140 & 49.50 & 48.95 \\
4 & 43560 & 45.2 & 10.7 & 4.034 & 5.568 & 8.971 & -5.805 & 0.8 & 2950 & 49.31 & 48.72 \\
5 & 41010 & 37.7 & 10.0 & 4.014 & 5.404 & 9.046 & -6.072 & 0.8 & 2850 & 49.08 & 48.44 \\
6 & 38450 & 30.8 & 9.3 & 3.989 & 5.229 & 9.314 & -6.369 & 0.8 & 2720 & 48.82 & 48.05 \\
7 & 35900 & 25.4 & 8.8 & 3.954 & 5.062 & 9.712 & -6.674 & 0.8 & 2570 & 48.51 & 47.43 \\
8 & 33340 & 21.2 & 8.3 & 3.926 & 4.883 & 9.934 & -7.038 & 0.8 & 2450 & 48.06 & 46.07 \\
\hline
\end{tabular}

type than that obtained from the ionization structure (Morisset et al. 2002). This seems to be a general problem and may be related to the metallicity of the region. Indeed, there is a loose correlation between the ionization structure - as measured by for example the $[\mathrm{Ne}$ III]/[Ne II] 15.5/12.8 $\mu \mathrm{m}$ line ratio - and the metallicity of H II regions (see Martín-Hernández et al. $2002 b$ ). Such a correlation may reflect the effects of metallicity on line blanketing or on the characteristics of the stellar wind.

Much theoretical effort has been dedicated to best describe the EUV spectra of massive stars. This is a formidable task because these stars have strong winds and extended atmospheres. This leads to strong non-LTE effects in the formation of spectral lines. Over the last ten years much progress has been made and current models include the effects of tens of thousands of lines on the energy balance and temperature structure of the stellar photosphere and wind. So far relatively modest effort has been investigated in systematic studies of the effects of metallicity on the stellar spectral energy distribution. In particular, there is no good theoretical understanding of the effects of metallicity on the ionizing fluxes of massive stars or on the optical and near-IR spectral characteristics used to type these stars. Here, we study the influence of metallicity on the spectral energy distribution of $\mathrm{O}$ stars and determine its influence on the resulting ionization structure of $\mathrm{H}$ II regions.

This paper is organized as follows. Section 2 presents a set of main-sequence (dwarf) star models constructed using the CMFGEN code by Hillier \& Miller (1998) and compares the predicted EUV fluxes with those from other codes. Section 3 presents a detailed analysis of the variations of the EUV spectral appareance and ionizing fluxes with effective temperature and metallicity. Section 4 investigates the influence of metallicity and other stellar parameters on the optical and near-IR spectral calibration. In Sect. 5, the ionizing structure of single star H II regions is studied. Finally, Sect. 6 presents the conclusions of this study.

\section{Description of the models}

The models presented in this paper are constructed using the CMFGEN program of Hillier \& Miller (1998), to which we refer for a full description of techniques. In short: CMFGEN iteratively solves the equations of radiative transfer subject to the constraints of statistical and radiative equilibrium, for an atmosphere with an outflowing stellar wind. The ions included in the non-LTE calculation are H I, He I-II, C IV, N III-V, O IV-VI, $\mathrm{Si} I V$ and Fe IV-VII. The cumulative effect of the iron lines, causing a line blanketing effect, is self-consistently accounted for by employing a description of the atomic models in terms of super levels. All and all, approximately 9000 transitions are accounted for.

We used this code to calculate a grid consisting of eight main-sequence (dwarf) star models ranging in effective temperature from $33000 \mathrm{~K}$ up to $51000 \mathrm{~K}$. Basic stellar parameters, $T_{\text {eff }}, R_{*}$ and $M_{*}$, using masses predicted by evolution theory, are taken from the Vacca et al. (1996) calibration and are given in Table 1.

We have opted to describe the photospheric density structure using a constant scaleheight

$H=\frac{k T_{\mathrm{eff}}}{\mu m_{\mathrm{amu}} g_{\mathrm{eff}}}$,

where $\mu$ is the mean molecular weight in atomic mass units, $g_{\text {eff }}$ is the effective gravity at the stellar surface corrected for radiation pressure by electron scattering and all other parameters have their usual meaning. Near and beyond the sonic point, the density is set by the velocity law through the equation of mass-continuity. The connection between photosphere and wind is smooth. The wind velocity structure is given by a standard $\beta$-law adopting $\beta=0.8$, which is representative for dwarf O stars (Groenewegen \& Lamers 1989). The terminal flow velocity $v_{\infty}$ follows from a scaling with the escape velocity (Lamers et al. 1995) and is also listed in Table 1. The mass-loss rates are from predictions by Vink et al. $(2000,2001)$. These authors show that for O-type stars the mass loss scales with the metal content $Z$ as

$\dot{M} \propto Z^{0.85}$.

Leitherer et al. (1992) showed that the final wind velocity also depends on metallicity as $v_{\infty} \propto Z^{0.13}$. We do not incorporate this dependence in the models. The microturbulent velocity was set to $20 \mathrm{~km} \mathrm{~s}^{-1}$ for the atmospheric structure calculations. For the calculation of the emergent spectrum we assumed microturbulent velocities of 20,10 and $5 \mathrm{~km} \mathrm{~s}^{-1}$. 
Table 2. Chemical composition adopted for the models with $Z=Z_{\odot}$.

\begin{tabular}{cc}
\hline \hline Element & Mass fraction \\
\hline $\mathrm{H}$ & 0.7023 \\
$\mathrm{He}$ & 0.2820 \\
$\mathrm{C}$ & $3.050 \times 10^{-3}$ \\
$\mathrm{~N}$ & $1.100 \times 10^{-3}$ \\
$\mathrm{O}$ & $9.540 \times 10^{-3}$ \\
$\mathrm{Si}$ & $6.990 \times 10^{-4}$ \\
$\mathrm{Fe}$ & $1.360 \times 10^{-3}$ \\
\hline
\end{tabular}

Table 3. Variation of the scaleheight and the mass loss rate with metallicity for models \#2 and \#5.

\begin{tabular}{cccccc}
\hline \hline & \multicolumn{2}{c}{ Model \#2 } & & \multicolumn{2}{c}{ Model \#5 } \\
\cline { 2 - 3 } \cline { 5 - 6 } & $H_{\star}$ & $\log \dot{M}$ & & $H_{\star}$ & $\log \dot{M}$ \\
$\mathrm{Z} / \mathrm{Z}_{\odot}$ & $\left(10^{-4} R_{\star}\right)$ & $\left(M_{\odot} / \mathrm{yr}\right)$ & & $\left(10^{-4} R_{\star}\right)$ & $\left(M_{\odot} / \mathrm{yr}\right)$ \\
\hline 2 & 7.817 & -5.120 & & 8.511 & -5.815 \\
$1 / 2$ & 8.554 & -5.631 & & 9.314 & -6.328 \\
$1 / 5$ & 8.702 & -5.971 & & 9.474 & -6.665 \\
$1 / 10$ & 8.751 & -6.225 & & 9.528 & -6.921 \\
\hline
\end{tabular}

In order to investigate the spectral appearance as a function of metallicity, we have calculated an additional set of models for our models \#2 and \#5, in which we set the metal content to $Z=2,1 / 2,1 / 5$ and $1 / 10 Z_{\odot}$. In conjunction with $Z$, we adjusted the helium abundance $Y$ according to

$Y=Y_{\mathrm{p}}+\left(\frac{\Delta Y}{\Delta Z}\right) Z$

where $Y_{\mathrm{p}}=0.24$ is the primordial helium abundance (Audouze $1987)$ and $(\Delta Y / \Delta Z)=3$ is an observed constant (Pagel 1992). To arrive at the appropriate metal abundances, we simply scaled the solar values (Cox 2000), which we give in Table 2 for reference. The mass-loss rates and scaleheights, which also depend on $Z$ through the mean molecular weight, of the additional models are given in Table 3.

\subsection{The assumption of a constant scaleheight}

The assumption of a constant scaleheight could lead to an overestimation of the effective gravity as derived from density sensitive lines. To test the validity of this assumption we recalculated model \#6 using an atmospheric structure calculated with the ISA-WIND code of de Koter et al. (1993, 1997). This provides a more realistic density stratification as it accounts for the effects of continuum radiation pressure on the atmosphere (see Sect. 2.2). For the comparison we focused on the spectral signatures investigated in this paper, i.e. the EUV, the optical He I $\lambda 4471$ and He II $\lambda 4542$ lines and the photospheric lines in the near-IR $K$-band.

Close inspection of the predicted EUV spectra shows that the number of ionizing photons below a certain wavelength, as well as the total amount of ionizing photons in the hydrogen and helium continua, agree within a few percent. The changes in the optical He I and He II lines are also negligible. A decrease of at most $3 \%$ in equivalent width is observed compared to the constant scaleheight model.

A fair comparison for the near-IR lines is more difficult, as many of these lines are partly formed in the transition zone where the photosphere connects to the stellar wind. In both the ISA-WIND as in the constant scaleheight model this connection is made in an ad hoc fashion. Keeping this in mind, we find that the differences in equivalent width between the two models is at most $4 \%$ for the $\mathrm{He}$ I lines and at most $12 \%$ for the $\mathrm{C}$ IV lines.

\subsection{Comparison with other model atmosphere codes}

Before comparing ionizing fluxes predicted by our models with those from other codes, we first briefly discuss the basic physics treated in the model atmospheres used for this comparison.

We focus on a comparison of main sequence (dwarf) O star models computed using the CMFGEN code (this study), the WM-BASIC code of Pauldrach et al. (2001) and the COSTAR models of Schaerer \& de Koter (1997). The latter make use of the ISA-WIND code of de Koter et al. (1993, 1997) to predict the stellar spectrum. A schematic comparison of assumptions made in these three codes is given in Table 4. Note that a comparison of codes does not address the question concerning the level of realism of the spectra produced. Assessing this issue requires empirical testing by analyzing $\mathrm{H}$ II regions containing single hot stars of which ideally the basic parameters have been determined from detailed quantitative spectroscopy using the same models as those applied to predict the ionizing fluxes. This approach has been followed by Crowther et al. (1999), who tested the CMFGEN and ISA-WIND models for a nebula containing a cool WN star and found similar results except for the ionizing flux distributions below the He I edge at $504 \AA$, which were quite different. The next best thing is to study $\mathrm{H}$ II regions containing single stars with well defined spectral types and effective temperatures. Schaerer (2000) reviews the success of non-LTE model atmospheres in reproducing diagnostic line ratios of nebula irradiated by O-type stars. The metallicity dependence of the ionizing flux may be studied by modelling extra-galactic $\mathrm{H}$ II regions (e.g. Bresolin et al. 1999; Oey et al. 2000; Bresolin \& Kennicutt 2002).

A comparison of ionizing fluxes from different codes is straightforward, however, interpreting the differences is - unfortunately - not. Modest discrepancies are expected to originate from small differences in the adopted abundances and photosphere and wind parameters of the models one is comparing. For instance, a difference in effective temperature of $1000 \mathrm{~K}$ yields a flux difference in the Wien part of the spectrum of $10-15 \%$. This may seem a needless "mistake" and one that is easily fixed. However, these complex models are difficult to compute and it turns out to be less straightforward than one may think to tune all parameters. Having said this, the main sources of differences between codes likely is in the way in which essential physical processes are treated. Notably $i$ ) the treatment of the equation of motion, $i$ ) the exact treatment of the non-LTE rate equations, iii) the number of lines included 
Table 4. Schematic comparison of assumptions made in the codes used for the EUV comparion. CMF implies a co-moving frame treatment of line transfer; SE denotes that the state of the gas is derived from statistical equilibrium (i.e. non-LTE). See text for a discussion.

\begin{tabular}{llll}
\hline \hline Treatment & CMFGEN & WM-BASIC & COSTAR \\
\hline $\begin{array}{l}\text { Temperature structure } \\
\text { Density structure }\end{array}$ & Consistent & Consistent & Grey-LTE with blanketing feedback \\
$\quad$ Photosphere & Constant H & Consistent & Grey-LTE $+p_{\mathrm{R}}$-cont \\
$\quad$ Wind & $\beta$-law & Consistent & $\beta$-law \\
Non-LTE treatment & Consistent & Consistent & Consistent (except Fe) \\
$\quad$ Line Transfer & CMF & CMF & Sobolev \\
$\quad$ Iron-group & SE & SE & Modified Nebular \\
Number of transitions/lines & & & \\
$\quad$ In SE & $\sim 9000$ & $\sim 30000$ & $\sim 1000$ \\
In spectrum & $\sim 17000$ & $\sim 4000000$ & $\sim 100000$ \\
Blanketing & Consistent & Consistent & Blanketing factors \\
\hline
\end{tabular}

(affecting the amount of line blocking), and iv) the way line blanketing effects are taken into account.

In the CMFGEN models we present here, we have described the photospheric density structure in a relatively simplistic way (see above). The CoSTAR models, using an atmospheric structure calculated with the ISA-WIND code, have a more sophisticated density stratification. Here a grey LTE temperature structure and an Euler equation taking into account continuum radiation pressure are iterated until convergence, accounting for the feedback of metal line blanketing on the Rosseland optical depth (see below). The wind is described in a similar way as for the CMFGEN models. The WM-BASIC models have the most self-consistent description of the density structure, as a final iteration is done, based on the non-LTE temperature structure, in which both the continuum and line force is included in the equation of motion. This yields the density structure throughout the photosphere and wind, i.e. no ad hoc wind velocity law is required.

The CMFGEN and WM-BASIC models compute the non-LTE state of the gas in a self-consistent way, using a super-level formalism. In total these codes treat $\sim 9000$ and $\sim 30000$ transitions, respectively. In this respect, the COSTAR models are relatively simple as the statistical equilibrium equations are solved explicitly for $\mathrm{H}, \mathrm{He}, \mathrm{CNO}$ and $\mathrm{Si}$ only, yielding $\sim 1000$ transitions, while the iron group elements, adding $\sim 100000$ lines, are treated in a modified nebular approximation. Moreover, the Sobolev approximation is applied to describe line transfer. Note that CMFGEN treats $\sim 17000$ individual lines in the emergent spectrum, while WM-BASIC accounts for more than 4000000 lines in the line-force and blocking calculations.

Line blanketing - i.e. redistribution of flux due to line opacity and the feedback of this opacity on the temperature structure as a result of the backwarming effect - is self-consistently treated in CMFGEN and WM-BASIC, but is only accounted for in an approximate way in the COSTAR code. To describe this in some detail: in COSTAR, a Monte-Carlo formalism describes the line transfer problem of the metals. On the one hand, this allows to describe the feedback of blocking on the atmospheric structure using wavelength averaged blocking factors. On the other hand, this neglects photon conversion. This "branching of photons" is relevant for redistributing flux, as it tends to "soften" the (EUV) radiation field.

The predicted EUV fluxes (especially in the He I continuum) are sensitive to line blanketing. In this respect, the CMFGEN and WM-BASIC models include a more consistent treatment and may in principle be expected to yield more reliable results. For the ionizing flux, the prescription of the wind structure is relatively unimportant for main sequence stars, as these have modest winds. The treatment of blanketing and the specification of the photospheric density structure affect the strengths of lines, and therefore may have an effect on He I and He II. As these lines are used to define spectral type, this may cause a modest uncertainty (up to one sub-type) in the spectral types assigned to the models. In this respect the WM-BASIC models are expected to behave best.

\section{The spectral energy distribution}

\subsection{Comparison of predicted EUV fluxes}

Figure 1 shows a comparison between the spectral energy distribution of comparable models computed with the three codes discussed above. The top left panel shows the emergent ionizing spectrum for our CMFGEN model for $T_{\text {eff }}=48670 \mathrm{~K}$ (model \#2). In the middle left panel, this CMFGEN model is shown together with WM-BASIC model D-50 $\left(T_{\mathrm{eff}}=50000 \mathrm{~K}\right.$, Pauldrach et al. 2001) and CoSTAR model C2 $\left(T_{\mathrm{eff}}=48529 \mathrm{~K}\right.$, Schaerer \& de Koter 1997). The bottom left panel shows partially integrated $q$ values of these three models. The spectra shown in the middel left panel are binned in wavelength to better bring out the overall flux behaviour, which is why they appear smooth. However, a forest of metal lines does contribute to all of them. The Lyman jump is modest due to the high degree of ionization. From $912 \AA$ down to the He I jump at $504 \AA$ (which is essentially absent), the most prominent features are strong resonance lines of $\mathrm{N}$ IV $\lambda 765$, O IV $\lambda 789, \lambda 609, \lambda 554$ and $\mathrm{OV} \lambda 629$. In this interval, the WM-BASIC model shows 

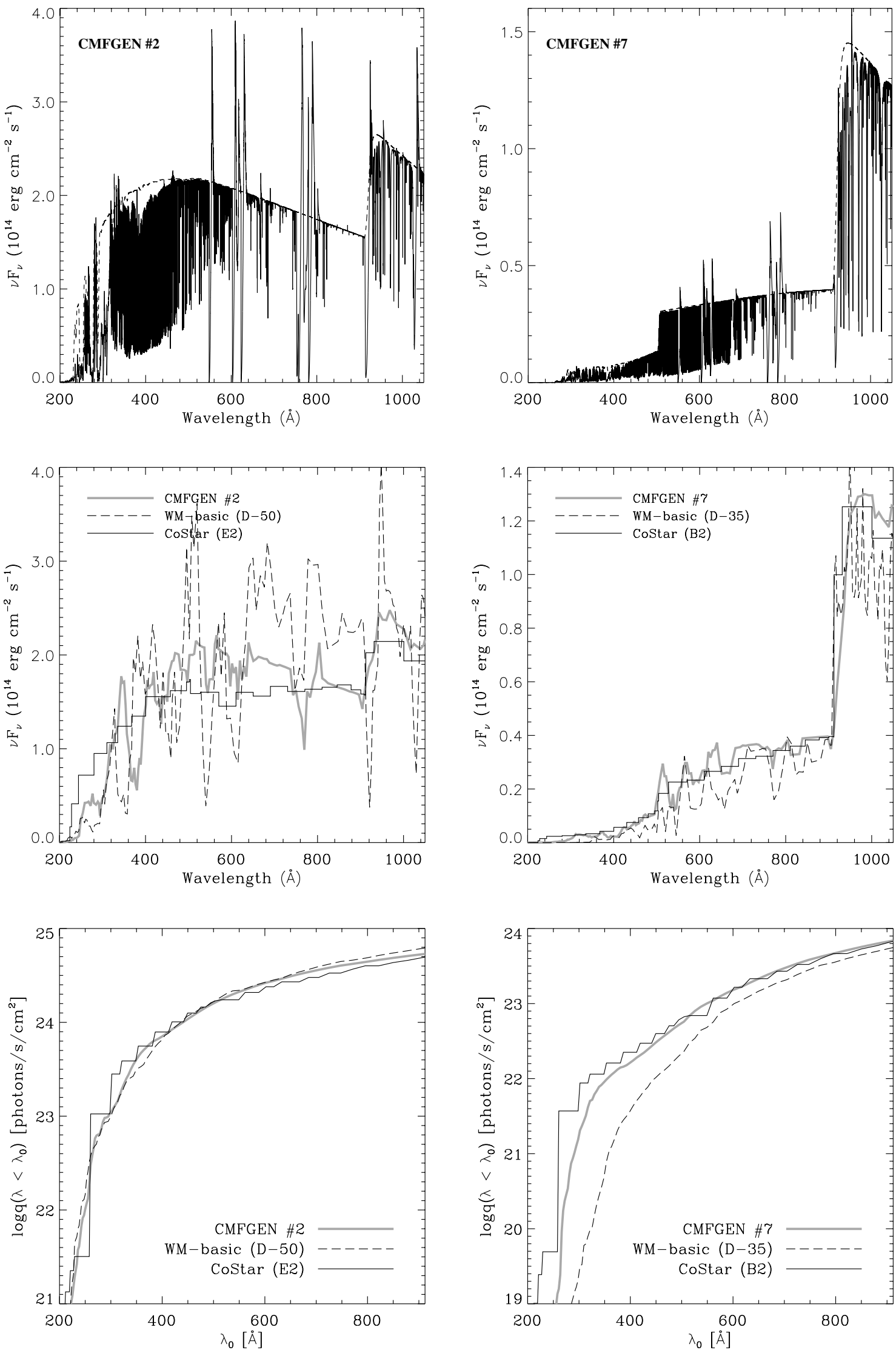

Fig. 1. Top panel: emergent SED for models \#2 $\left(T_{\mathrm{eff}}=48670 \mathrm{~K}\right)$ and $\# 7\left(T_{\mathrm{eff}}=35900 \mathrm{~K}\right)$. The continuum is plotted by a dashed line. Middle panel: comparison of the emergent SED for models \#2 (left panel) and \#7 (right panel) with CoSTAR and WM-BASIC dwarf models at similar $T_{\text {eff }}$. Bottom panel: the number of photons in $\mathrm{cm}^{-2} \mathrm{~s}^{-1}$ below wavelength $\lambda_{0}$ calculated from the models considered in the middle panel is plotted as a function of this wavelength $\lambda_{0}$. 
an on average about $15 \%$ higher flux level compared to our CMFGEN calculation. This can be explained by the temperature difference of the models. Taking $T_{\text {eff }}$ effects into account, the COSTAR model produces a somewhat softer spectrum.

In the He I continuum the spectrum is dominated by a dense forest of mostly Fe IV-VI lines. Here the CMFGEN and WM-BASIC model show a roughly similar behaviour, both in terms of continuum flux and amount of line blocking. The COSTAR model now shows a harder spectrum. The explanation for the COSTAR predictions likely involves the treatment of the blocking factors, which result from a Monte-Carlo simulation in which the effects of photon conversion (or "branching") are neglected. This forces all radiative excitation/deexcitation processes to be scatterings, which prevents flux to be distributed towards longer wavelengths and therefore keeps the radiation field "hard". This would also explain the relatively soft flux in the COSTAR spectrum at $\lambda \gtrsim 500 \AA$. Other effects that may play a (more modest) rôle are the temperature gradient in the He I continuum. A steeper gradient causes a higher flux at relatively short wavelengths (where the continuum is formed at relatively high Rosseland optical depth), while at relatively long wavelengths the flux will be lower. Also the neglect of line-broadening in COSTAR models may have an effect (see Schaerer \& de Koter 1997). Note that the exact location of the line forest differs between the CMFGEN and WM-BASIC models. (To see this properly one first has to correct the continuum levels for differences in $T_{\text {eff }}$.) This is most likely connected to differences in the predicted ionization of iron. The relevant iron ions each show a preferred region in which their line forest is concentrated. It holds very roughly that this is at 510-440 for Fe IV; 460-320 $\AA$ for Fe V; 340-260 $\AA$ for Fe VI, and at 270-160 for Fe VII. This suggests that the dominant photospheric ionization of the WM-BASIC model is Fe VI, while Fe V is most important in CMFGEN (see the pronounced difference at $\sim 400 \AA$ ).

Though not visible in the figure, the He II continuum fluxes of the CMFGEN and WM-BASIC models agree reasonably well (which may be slightly surprising in view of the difference in $\left.T_{\text {eff }}\right)$. Again the COSTAR model produces a harder spectrum. The same reasons as discussed above may apply. However, it may also be connected to the use of the Sobolev approximation and/or the velocity structure in the wind. The latter may cause a depopulation of the He II ground state as a result of the velocity gradient in the wind, in combination with the temperature in the continuum forming layers at the wavelength of the He II resonance lines (Gabler et al. 1989).

The right panels show a comparison between our CMFGEN model for $T_{\text {eff }}=35900 \mathrm{~K}$ (model \#7), WM-BASIC model D-35 having $T_{\text {eff }}=35000 \mathrm{~K}$, and CoSTAR model B2 for which the temperature is $36300 \mathrm{~K}$. Taking into account the differences in effective temperature, the EUV flux distributions compare well though again the Lyman flux at $\lambda \gtrsim 700 \AA$ of the COSTAR model is somewhat softer. Below $\sim 700 \AA-$ i.e. again in the regime of severe flux blocking - the opposite behaviour is seen: the COSTAR spectrum is harder. The likely reasons for this behaviour have been discussed above. A detailed inspection of the energy distributions shows that the WM-BASIC models produce a clear drop-off in flux at the $\mathrm{N}$ III and $\mathrm{C}$ III ionization edges near $260 \AA$. The presence of this sizable bound-free jump is somewhat surprising in view of the high $T_{\text {eff }}$ of the model, and is not seen in CMFGEN and COSTAR models.

The fluxes in the He II continua of the three models differ by orders of magnitude. Possible reasons for these discrepancies have been discussed above.

We conclude that down to $\sim 500 \AA$ the EUV flux distributions compare reasonably well, though the flux levels do differ. However, at shorter wavelengths large (order of magnitude) discrepancies may occur. For the CoSTAR predictions, in comparison to the other two codes, the major part of these differences can be traced back to the treatment of blanketing. The models also show large differences for the He II continuum fluxes. This is a relevant problem with respect to the internal consistency of different code predictions. However, in O-type stars the dominant flux component at $\lambda<228 \AA$ tends to be that of non-thermal soft X-ray emission arising from shocks in the stellar wind (e.g. MacFarlane et al. 1994; Feldmeier et al. 1998). As no self-consistent description of this process is presently available, this seems a more acute problem.

\subsection{Comparison of ionizing fluxes}

Figure 2 shows the total number of Lyman continuum photons emitted per second, $Q_{0}$, and the total number per second per $\mathrm{cm}^{2}, q_{0}=Q_{0} / 4 \pi R_{*}^{2}$, for both our O star main sequence models and the comparison models discussed above. Also given is the ratio between Lyman and He I continuum photons, $Q_{0} / Q_{1}$ (bottom panel). We added predictions from Kurucz models, presented by Vacca et al. (1996); however, we will not include these in the discussion of the results.

To eliminate differences in the adopted stellar radii, we first focus on the predictions for $q_{0}$. The results are plotted versus $T_{\text {eff }}$, allowing us to assess the differences in $q_{0}$ as a result of small differences in adopted temperatures. All predictions are very similar, only showing discrepancies up to $0.1 \mathrm{dex}$. Why is this so?

The principal answer is that though line blanketing effects are strong at wavelengths shorter than $\sim 500$ (700) $\AA$ in the case of model \#2 (\#7), the flux is mainly redistributed within the Lyman continuum. Only limited energy "leaks" out at $\lambda>912 \AA$. This may be illustrated using a simple estimate. The line forest removes $\sim 40 \%$ of the flux in both model examples. These photons are back-scattered and re-thermalized. Assuming the thermal emission may be characterized by the emerging continuum flux distribution, one expects $60 \%$ (20\%) of this energy to be emitted still within the Lyman continuum. Therefore, only $16 \%$ (32\%) leaks out into the Balmer continuum and beyond. In terms of number of ionizing photons, the "damage" is even less as redistribution of energy towards longer wavelengths within the Lyman continuum requires an increase in the number of photons. This explains why the number of ionizing photons only differ up to $\sim 0.05$ and $0.1 \mathrm{dex}$ for models \#2 and \#7 respectively. The neglect of branching in COSTAR models is separate from the above discussion (it concerns forward-scattered photons). However, note that the iron forest is dominated by transitions from meta-stable levels. 


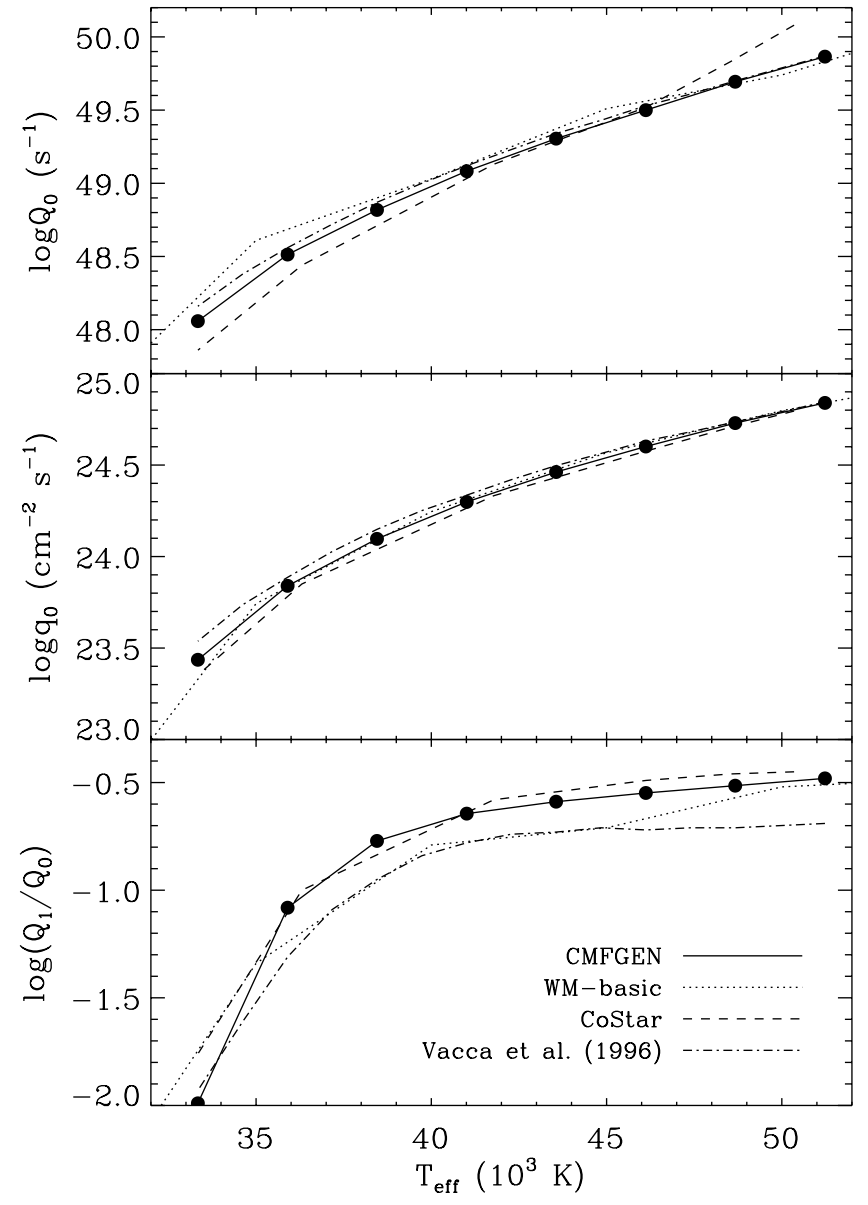

Fig. 2. Variation of the Lyman continuum ionizing photons $Q_{0}$ (top panel), $q_{0}$ (middle panel), and the hardness ratio $Q_{1} / Q_{0}$ (bottom panel) with effective temperature. The results from our CMFGEN models (solid line) are compared to those from the WM-BASIC models (dotted line), CoSTAR models (dashed line) and Vacca et al. (1996) (dash dotted). We use WM-BASIC models D-30, D-35, D-40, D-45, D-50 and D-55 (Pauldrach et al. 2001), and CoSTAR models A2, B2, C2, D2, E2 and F2 (Schaerer \& de Koter 1997).

The level structure of the relevant iron ions is such that branching typically produces two photons, one at $\lambda<912 \AA$ and one at $\lambda>912 \AA$. Therefore, also this effect preserves Lyman continuum photons, though it does affect the hardness ratio $Q_{1} / Q_{0}$. The former is illustrated in the bottom panels of Fig. 1, where we show partially integrated $q$ values.

The hardness ratio $Q_{0} / Q_{1}$, also independent of stellar radius, yields agreement within $\sim 0.2$ dex. The COSTAR models having $T_{\text {eff }}>40000 \mathrm{~K}$ show a harder spectrum than do the CMFGEN and WM-BASIC models - as discussed above. This is in agreement with the flux differences in the Lyman and He I continua discussed for model \#2.

The apparent reasonable agreement between number of ionizing photons is somewhat misleading. Nebular lines from ions such as Ne III or S IV require radiation from below $500 \AA$. In this spectral regime, as we have seen above, the ionizing fluxes may differ strongly implying large differences in emission line strength.
Table 5. Predicted number of ionizing photons $Q_{0}$ and $Q_{1}$ for \#2 and $\# 5$ as a function of metal abundance.

\begin{tabular}{cccccc}
\hline \hline & \multicolumn{2}{c}{ Model \#2 } & & \multicolumn{2}{c}{ Model \#5 } \\
\cline { 2 - 3 } \cline { 6 - 6 }$Z / Z_{\odot}$ & $\log Q_{0}$ & $\log Q_{1}$ & & $\log Q_{0}$ & $\log Q_{1}$ \\
\hline 2 & 49.70 & 49.16 & & 49.09 & 48.40 \\
1 & 49.69 & 49.18 & & 49.08 & 48.44 \\
$1 / 2$ & 49.69 & 49.19 & & 49.07 & 48.46 \\
$1 / 5$ & 49.68 & 49.20 & & 49.07 & 48.47 \\
$1 / 10$ & 49.67 & 49.21 & & 49.06 & 48.48 \\
\hline
\end{tabular}

\subsection{EUV fluxes and $Q_{0}$ and $Q_{1}$ values as a function of metal content}

In Table 5 we show the predicted number of ionizing photons $Q_{0}$ and $Q_{1}$ for models \#2 and \#5 as a function of metal abundance. The results show that a decrease in $Z$ of a factor of twenty only alters the number of Lyman continuum photons by 0.03 dex. Over the same metallicity range the number of He I continuum photons increases by up to 0.08 dex.

The modest dependence on metal content is again the result of the way line blanketing redistributes the flux (see above). In the top left panel of Fig. 3 we show a comparison of the EUV spectrum of solar abundance model \#5 with that of a model with identical basic parameters, only with $Z=1 / 10 Z_{\odot}$; in the top right panel the solar and twice solar model are compared. Relative to the $1 / 10 Z_{\odot}$ model, the solar metallicity prediction clearly shows an increased flux level in the region from the Lyman jump down to $\sim 600 \AA$, which is compensated by a decreased flux below $600 \AA$. This is best seen in the bottom left panel, where we show binned versions of the spectra. The tendency to redistribute flux within the Lyman continuum is also illustrated in Fig. 4, where we show partially integrated $q$ values.

The conservation of ionizing photons works less well for $Q_{1}$, as expected. Here a decrease in metallicity of a factor twenty results in up to $20 \%$ more ionizing photons. This implies that those nebular species that depend on a specific EUV flux range below $\sim 500 \AA$ may show a strong dependence on metallicity. We will discuss this in Sect. 5.

The results for the number of hydrogen ionizing photons are similar to those obtained by Smith et al. (2002) using unified WM-BASIC models, by Lanz \& Hubeny (2003) using plane parallel TLUSTY models and by Kudritzki (2002), who used WM-BASIC models of very massive $O$ stars at $Z=10^{-4} Z_{\odot}$ to $Z=Z_{\odot}$. The results of the two former studies show that the convervation of $Q_{0}$ for dwarf stars breaks down at lower effective temperatures (at 32000 to $37500 \mathrm{~K}$ depending on model assumptions). Compared to our models the number of He I continuum photons in the models of Smith et al. do not show a similar behaviour. The models of Kudritzki and Lanz \& Hubeny do show a conservation of the number of photons, where the latter models again show that this convervation breaks down for lower effective temperatures $(\$ 45000 \mathrm{~K})$. 

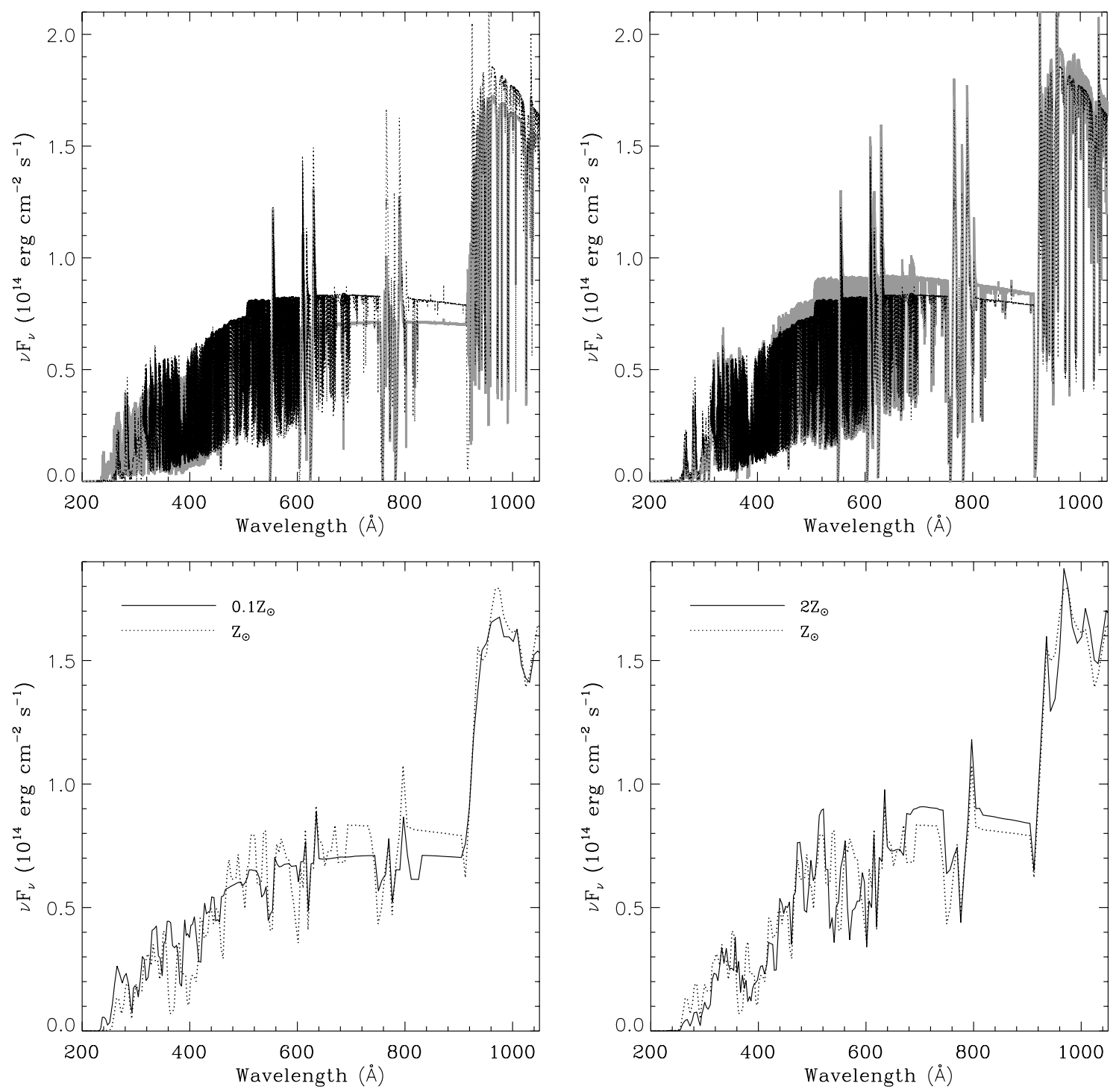

Fig. 3. Top panels: comparison of the EUV spectrum of solar abundance model \#5 (dotted line) with equivalent models with $Z=1 / 10 Z_{\odot}($ left panel) and $Z=2 Z_{\odot}$ (right panel), plotted in grey. Bottom panels: binned version of the spectra plotted in the top panel.

\section{Spectral classification}

\subsection{Optical classification}

The optical spectral classification of $\mathrm{O}$ stars relies on a quantitative criterion based on the ratio of the equivalent widths of HeI $\lambda 4471$ and He II $\lambda 4542$ (Conti \& Alschuler 1971; Conti \& Frost 1977; Mathys 1988). In O9 stars, the HeI $\lambda 4471$ line is stronger; the ratio changes gradually so that the He II $\lambda 4542$ line begins to dominate at type $\mathrm{O} 6$ and earlier. According to this criterion, the value of $\log \left[W_{\lambda}(4471) / W_{\lambda}(4542)\right]$ unambiguously determines the spectral type of the star.

This observational criterion can be used to assign spectral types to our models. Figure 5 compares the equivalent widths of the He I and He II classification lines observed in dwarf stars with the predictions from our models. The slopes of the dotted lines correspond to the ratios of the equivalent widths delimiting the $\mathrm{O}$ star subclasses according to the calibration of
Mathys (1988). We investigate the assigned spectral classification for the effects of varying respectively $\log g$, metallicity and microturbulent velocity. Before discussing these dependencies in detail, we will first elaborate on how well our initial model grid for dwarf stars fits these observations.

The observational data for the dwarf stars are taken from Conti \& Alschuler (1971), Conti (1973), Conti \& Frost (1977), and Mathys (1988, 1989). The luminosity class was verified using the spectral classification of Walborn (1971, 1972, 1976, 1973, 1982). Stars that were not classified as dwarfs by Walborn were discarded. Spectroscopic binaries were identified using the catalogue of Pedoussaut et al. (1996) and stars with variable radial velocity using Mathys $(1988,1989)$. These were also removed. Finally, variable stars were deselected using the catalogue of Kholopov et al. (1998). In the top left panel of Fig. 5 we show the selected observed data (using open diamond symbols), including the error bars. The observations cover a strip in the He I $\lambda 4471$ versus He II $\lambda 4542$ equivalent width diagram that is broader than can be accounted for by 


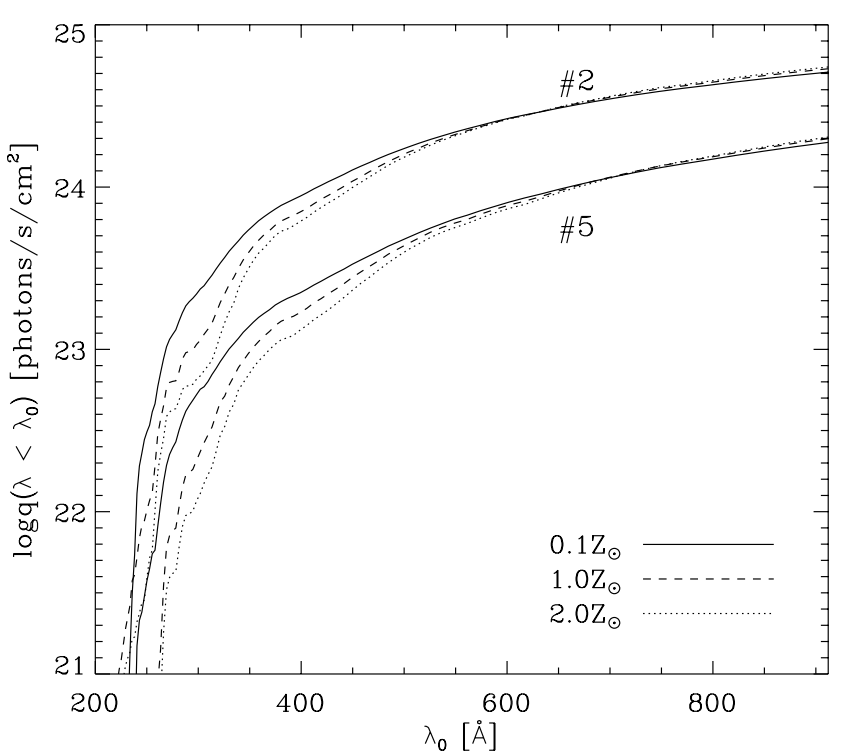

Fig. 4. The effect of the metal content on the number of photons in $\mathrm{cm}^{-2} \mathrm{~s}^{-1}$ below a certain wavelength $\lambda_{0}$ is plotted as a function of this wavelength $\lambda_{0}$. The effect is shown for models \#2 and \#5.

uncertainties in individual measurements. The width of this strip therefore also reflects differences in stellar properties such as gravity, mass loss, metallicity and/or photospheric properties such as microturbulent velocity.

\subsubsection{Mass loss}

Also plotted in the top left panel are the models listed in Table 1. The predictions reproduce the observed range of He I $\lambda 4471$ equivalent width values well. The equivalent width of He II $\lambda 4542$ seems to be overestimated by approximately 10 to $20 \%$ for early- and mid-O spectral types. A possible explanation for this overprediction may be an adopted mass loss that is systematically too low. A higher mass loss would reduce the equivalent width, as wind emission fills in more of the photospheric absorption profile (Lanz et al. 1996). This effect is especially important for the earliest spectral types, as these have the strongest winds. The top right panel of Fig. 5 shows the effect of an increase in $\dot{M}$. Here a grid with doubled mass loss rate, indicated by squares, is compared to the standard grid (circles). A change of this magnitude is characteristic of the typical error in the comparison of mass-loss determinations (Puls et al. 1996) and mass-loss predictions (Vink et al. 2000). The line strength of He II $\lambda 4542$ is decreased by up to 10 to $20 \%$ in the early type models, as a result of wind emission. A modest decrease in the He I $\lambda 4471$ strength is also observed.

\subsubsection{Gravity}

The width of the observed strip of equivalent width ratios is partly explained by gravity effects. Kudritzki et al. (1983) already showed that the ratio $W_{\lambda}(4471) / W_{\lambda}(4542)$ depends on luminosity class. Here we show that gravity variations within a luminosity class also significantly effect this ratio. The main sequence gravities adopted by Vacca et al. (1996) should be seen as mean values for the ensemble of stars of given spectral type used for their calibration. We have calculated a grid of models in which we increased and decreased the stellar gravity by 0.3 dex. This roughly corresponds to the typical difference between dwarf and giant stars. The bottom left panel of Fig. 5 shows the predicted strip of equivalent width ratios spanned by this gravity range. Note that e.g. a decrease (increase) of $\log g$ by 0.3 dex implies about one spectral sub-type earlier (later). A decrease of $\log g$ implies a decrease in electron density, which causes a decrease in the recombination rate, effectively increasing the helium ionization. The opposite is true in case of an increased gravity.

The new temperature scale of O stars (Martins et al. 2002; see also de Koter et al. 1998) implies a decrease in $T_{\text {eff }}$ of about 10 percent compared to the calibration of Vacca et al. (1996) that is used for our grid. The lower luminosity and mass implied by this new $T_{\text {eff }}$ results, however, in only a modest shift of $\sim 0.06$ dex in gravity. Therefore, this temperature recalibration can only account for a very minor part of the overprediction of He II $\lambda 4542$.

In part the overprediction of He II $\lambda 4542$ may also be the result of a to high stellar mass. Indeed, a systematic difference has been noted in masses that are based on spectroscopic analysis of Balmer line profiles an those that result from a comparison of basic parameters ( $L_{*}$ and $T_{\text {eff }}$ ) with evolutionary tracks (e.g. Herrero et al. 1992). Recent new results (Herrero et al. 2002) appear to show that most of this discrepancy can be explained by improved spectroscopic modeling and the lower $T_{\text {eff }}$ values for O-stars. Still, adopting the spectroscopic masses as presented by Vacca et al. (1996) (which are 30 to $70 \%$ less for the earliest spectral types) yields a decrease in He II $\lambda 4542$ equivalent width of about 10 to $15 \%$ for the earliest spectral types. This should be seen as an upper limit of a possible mass effect.

\subsubsection{Metallicity}

Metallicity effects may also explain part of the scatter in equivalent widths, but are not expected to be responsible for the systematic shift in the He II $\lambda 4542$ line strength. Variations in the stellar metallicity are undoubtedly present in Galactic $\mathrm{O}$ stars. Emission-line studies of $\mathrm{H}$ II regions, planetary nebulae and supernova remnants consistently show that the average abundance of oxygen decreases with Galactocentric distance by $\sim 0.06$ dex per kpc, with values of $\sim 2.5 Z_{\odot}$ in the Galactic Center, and down to $\sim 1 / 4 Z_{\odot}$ at Galactocentric distances larger than $15 \mathrm{kpc}$ (see e.g. Henry \& Worthey 1999). Low abundance systems such as the Small Magellanic Cloud have metallicities of the order of $\sim 1 / 8 Z_{\odot}$. The top right panel in Fig. 5 shows the variation of the equivalent widths of He I $\lambda 4471$ and He II $\lambda 4542$ as a result of changing the atmospheric metallicity - and its indirect effect on line blanketing and stellar wind mass loss - from $1 / 10$ to $2 Z_{\odot}$. The models with modified metal content are indicated by solid triangles.

Two effects related to metal content may influence the line strength of the He I $\lambda 4471$ and He II $\lambda 4542$. First, an increased metallicity implies an increased line blanketing and therefore stronger diffuse field. This will increase the ionization of 


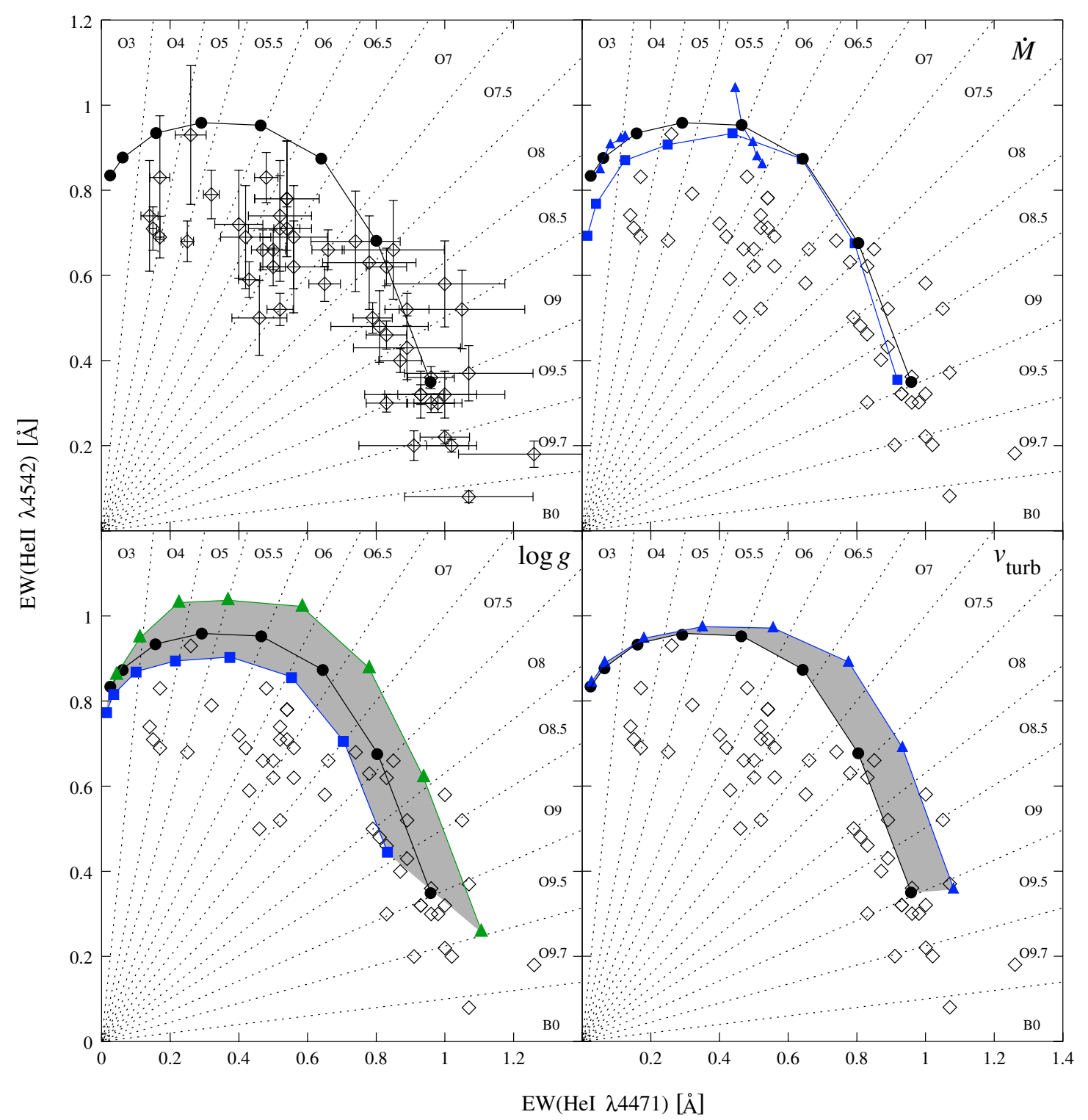

Fig. 5. Top left panel compares the equivalent width of He I $\lambda 4471$ and He II $\lambda 4542$ measured in the model grid with observed values in main sequence (dwarf) stars (open diamonds). Bottom left panel shows the effects of an increase (triangles) and a decrease (squares) of 0.3 dex in $\log g$. In the top right panel a grid with doubled mass-loss (squares) is compared to the model grid (circles). Also shown are the equivalent widths of models \#2 and \#5 with $Z=2,1 / 2,1 / 5$ and $1 / 10 Z_{\odot}$ (triangles). The decrease in metallicity results in an increase of the equivalent width of He I $4471 \AA$. The bottom right panel compares a grid with $v_{\text {turb }}=5 \mathrm{~km} \mathrm{~s}^{-1}$ (circles) to a grid with $v_{\text {turb }}=20 \mathrm{~km} \mathrm{~s}$ (triangles). The dotted lines indicate the parameter space delimited by every subclass according to the empirical classification by Mathys (1988).

helium. The top right panel of Fig. 5 indeed shows this behaviour for model \#5, i.e. a decrease in the He I and an increase in the He II line strength. Second, an increased metallicity implies a stronger stellar wind, therefore an increased filling in of line profiles by wind emission (see also e.g. Herrero 2003). This effect is more important for earlier type stars, which have a larger mass loss. This second effect dominates in model \#2, resulting in a decreased He II $\lambda 4542$ line strength with increased metallicity and vice versa.

We conclude that metallicity effects may change the spectral type by up to one subtype. This is in agreement with findings of Puls et al. (2003), which showed that the inclusion of line blanketing at SMC metallicities resulted in a much more modest change in $T_{\text {eff }}$ relative to that found for Galactic stars.

\subsubsection{Microturbulence}

The effect of microturbulence is expected to explain part of the scatter in the observed He I $\lambda 4471$ and He II $\lambda 4542$ equivalent width ratios and is shown in the bottom right panel of Fig. 5. The standard grid assumes a constant microturbulent velocity of $5 \mathrm{~km} \mathrm{~s}^{-1}$. We calculated a new grid adopting $v_{\text {turb }}=$ $20 \mathrm{~km} \mathrm{~s}^{-1}$, producing stronger lines. A comparison shows that the He II line is almost insensitive to the introduced variation in microturbulent velocity. The saturated He I line however shows a significant change in strength for models of spectral type O5 and later. The relative changes are $4 \%$ for the model with the weakest $\mathrm{HeI}$ line and $12 \%$ to $20 \%$ for the models with the strongest He I lines. An increased microturbulence may shift 


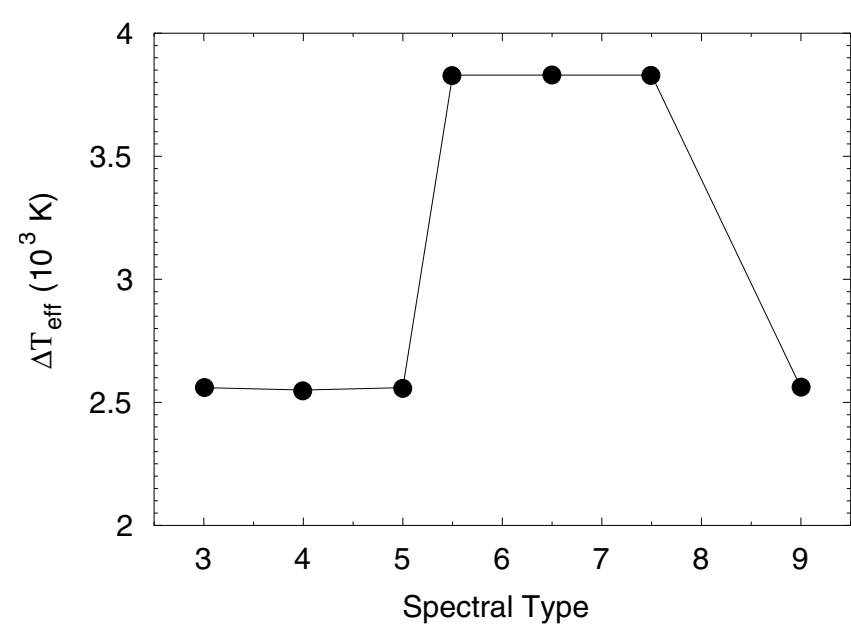

Fig. 6. Effective temperature shift with spectral type between the Vacca et al. (1996) calibration and the temperature scale derived from the models with $Z=Z_{\odot}$ and $v_{\text {turb }}=5 \mathrm{~km} \mathrm{~s}^{-1}$.

the assigned spectral type by up to half a sub-type towards later spectral type. The impact of microturbulence in the line formation calculations of $\mathrm{H}$ and $\mathrm{He} \mathrm{I}$ in $\mathrm{OB}$ stars, and consequently, in the derived stellar atmospheric parameters, has been investigated by several authors (e.g. Smith \& Howarth 1998; McErlean et al. 1998; Villamariz \& Herrero 2000).

Summing up the above discussion, we conclude that all-in-all the predicted equivalent width of $\mathrm{He}$ I $\lambda 4471$ and He II $\lambda 4542$ reproduce the observed behaviour reasonably well, though the He II line strength is somewhat overpredicted for early- and mid-O types. We also show that the spectral type of dwarf stars is not only a function of effective temperature of the star, but also depends, albeit to a lower degree, on microturbulent velocity, metal content and on gravity, i.e. gravity variations within the dwarf class. Variations in these parameters explain the observed strip of equivalent width of $\mathrm{He}$ I $\lambda 4471$ and He II $\lambda 4542$ for given spectral type.

Finally, when comparing the spectral types of our models to the original spectral types assigned by Vacca et al. (1996), we see that there is a systematic shift to earlier spectral types for given temperature. In other words, the $T_{\text {eff }}$ scale derived with our models shows a systematic shift to lower temperatures. Figure 6 shows the difference for $Z=Z_{\odot}$ and $v_{\text {turb }}=5 \mathrm{~km} \mathrm{~s}^{-1}$. The decrease from the Vacca et al. scale to our new scale varies between $\sim 2500 \mathrm{~K}$ and $\sim 3800 \mathrm{~K}$ and is largest for stars in the spectral range of $\mathrm{O} 5$ to $\mathrm{O} 9$. This decrease of $T_{\text {eff }}$ due to line blanketing effects has recently been found in several studies (e.g. de Koter et al. 1998; Crowther et al. 2001; Martins et al. 2002).

\subsection{K-band classification}

$K$-band $(2 \mu \mathrm{m})$ spectroscopy has been used in the past few years to identify and classify the characteristics of newlyformed $\mathrm{O}$ stars that are found in heavily obscured $\mathrm{H}$ II regions (e.g. Watson et al. 1997; Watson \& Hanson 1997; Kaper et al. 2002; Bik et al. 2003; Martín-Hernández et al. 2003). The typical dust extinction of more than $10 \mathrm{mag}$ at visual wavelengths

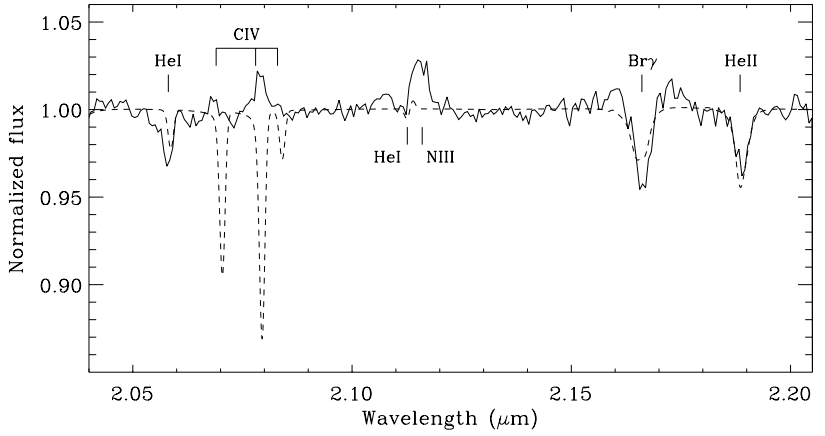

Fig. 7. The synthetic $K$-band spectrum from model \#4 with $Z=Z_{\odot}$ and $v_{\text {turb }}=10 \mathrm{~km} \mathrm{~s}^{-1}$, which corresponds to an $\mathrm{O} 5$ main sequence star (dashed line) is compared to the spectrum of the $\mathrm{O} 5 \mathrm{~V}((\mathrm{f}))$ star HD 93204 (solid line). The synthetic spectrum is degraded to the resolution of the observed spectrum $(\lambda / \Delta \lambda \sim 1500)$. The lines observed in the $K$-band are indicated. Note that while the $\mathrm{C}$ IV doublet transition is clearly observed in emission, our model predicts it to be in absorption. The N III emission at $2.116 \mu \mathrm{m}$ is not included in the models.

towards these $\mathrm{H}$ II regions has restrained optical photometry and spectroscopy of such stars. At near infrared wavelengths the extinction is much less, and direct observations of their photospheres are possible. At longer wavelengths observations fail to detect their photosphere directly because the spectral energy distribution is dominated by emission from the surrounding dust. Hence, it becomes essential to understand the behaviour of the photospheric features in the near-IR regime, the only observational window of such young $\mathrm{O}$ stars. In this section we compare the $K$-band spectral behaviour of our models with observations.

Hanson et al. (1996) present an atlas of $K$-band spectra of a large number of relatively nearby, well-studied, optically visible massive stars with the intention of investigating the variation of $2 \mu \mathrm{m}$ spectral features with spectral type and luminosity in known OB stars. They find that in early-O dwarf stars (O3, O4, some O5), He II $\lambda 2.189$ (wavelengths of the near-IR lines are in micrometers) absorption and N III (observed at $2.116 \mu \mathrm{m}$ ) emission are the dominant spectral indicators. Mid-O stars (O5, O6, some O7) show C IV 12.078 emission and can begin to show He I $\lambda 2.113$ absorption. Late-O (O8, O9) and early-B stars lack He II, N III and CIV, showing instead He I and fairly strong (in comparison to hotter O stars) HI $\lambda 2.1661$ and Brackett $\gamma$.

Our grid of models can be used as a first test against the features found in the $K$-band spectrum of O-type dwarf stars. Many complications are to be expected in the modeling of these lines since they are formed in the transition region from the stellar photosphere to the super-sonic stellar wind (see e.g. Kudritzki \& Puls 2000). A full parameter study is highly desirable to understand their diagnostic value. In this paper, we only discuss the temperature and microturbulent velocity effects and refer to Lenorzer et al. (in prep.) for a detailed parameter study.

Figure 7 shows, as an example, the comparison between the synthetic $K$-band spectrum from model \#4 with $Z=Z_{\odot}$ and $v_{\text {turb }}=10 \mathrm{~km} \mathrm{~s}^{-1}$ (which corresponds to an O5 main sequence star) and the spectrum of HD 93204, an O5V((f)) star observed by Hanson et al. (1996). The synthetic spectrum was 
degraded to the resolution of the observed spectrum $(\lambda / \Delta \lambda \sim$ 1500). The HeI lines at 2.058 and $2.113 \mu \mathrm{m}, \mathrm{Br} \gamma$, and the $\mathrm{He}$ II line at $2.189 \mu \mathrm{m}$ are clearly visible in the synthetic spectrum. Qualitatively, the behaviour of these lines closely resembles that shown in the $2 \mu \mathrm{m}$ atlas. He I $\lambda 2.058$ is almost undetectable in our three hottest models but starts to show a strong absorption in the intermediate temperature models to finally weaken in model \#8. Hanson et al. (1996) observed that this line may turn into emission for B stars. Br $\gamma$ and He I $\lambda 2.113$ strengthen towards later-type stars, while He II $\lambda 2.189$ becomes progressively weaker.

The models show a discrepant behaviour in the case of the metal lines. The N III emission at $2.116 \mu \mathrm{m}$, with transition $8-7$, is simply not included in the models. The C IV doublet transition $\left(3 \mathrm{pP}^{0}-3 \mathrm{~d}^{2} \mathrm{D}\right)$, observed in emission $\mathrm{O} 4$ to O8.5 stars (Hanson et al. 1996), is accounted for in our models. It is, however, predicted in absorption in all models that have $T_{\text {eff }} \gtrsim 41000 \mathrm{~K}$, i.e. spectral type $\mathrm{O} 5$ or earlier. The disappearance of these lines in late O-type stars is well predicted and is the result of the recombination of C IV to C III. The origin of the observed emission lines is more difficult to understand. Recent high spectral resolution $(R \sim 8000)$ observations of O-type stars (Bik et al. in prep.) resolve these C IV lines in a few stars, yielding a $F W H M$ of about $40 \mathrm{~km} \mathrm{~s}^{-1}$. Moreover, the strength of the lines (e.g. Br $\gamma$ ) as observed by Hanson et al. is not found to be much stronger in supergiants than in dwarf stars. These arguments strongly suggest that the near-infrared $\mathrm{C}$ IV lines have a predominantly photospheric origin, therefore their emission cannot be attributed to an enhanced C IV abundance and/or wind effects. If the emission was due to a temperature inversion in the line forming region, this would similarily affect He I 22.058. Indeed, both lines have regions of formation that largely overlap such that a temperature inversion would also be visible in the He I line. Our models, however, reproduce the He I line reasonably well. The transition of C IV observed in the near-infrared spectrum of O-stars involves low atomic levels that are populated via transitions located in the UV and far-UV. It is therefore likely that we do not reproduce the behaviour of these lines due to a mistreatment of this complex spectral region. This issue is still being investigated but is encouraged by recent calculations including $\mathrm{C}$ III which show the $\mathrm{C}$ IV doublet transition in emission. We therefore suspect that line pumping is the cause of these emission lines (Lenorzer et al. in prep.)

We carried out a quantitative comparison with observed equivalent widths using the $\mathrm{He}$ I $\lambda 2.058, \mathrm{Br} \gamma$ and $\mathrm{He}$ II $\lambda 2.189$. Figure 8 compares the predicted equivalent widths of these three lines adopting $v_{\text {turb }}=5,10$ and $20 \mathrm{~km} \mathrm{~s}^{-1}$, with those measured by Hanson et al. (1996). Spectral types of the models are those derived from the optical lines (see Fig. 5). The microturbulent velocity has no significant effect on the lines. The models fit reasonably well the observed equivalent widths, although they overestimate the strength of He II $\lambda 2.189$ for spectral types $\mathrm{O} 3$ and $\mathrm{O} 4$.

We can investigate whether the equivalent widths of the $2.058 \mu \mathrm{m} \mathrm{He}$ I, Br $\gamma$ and $2.189 \mu \mathrm{m}$ He II lines are affected by variations in the metal content. For model \#2 with $v_{\text {turb }}=$ $10 \mathrm{~km} \mathrm{~s}^{-1}$ (which corresponds to an $\mathrm{O} 3 \mathrm{star}$ ), a change in

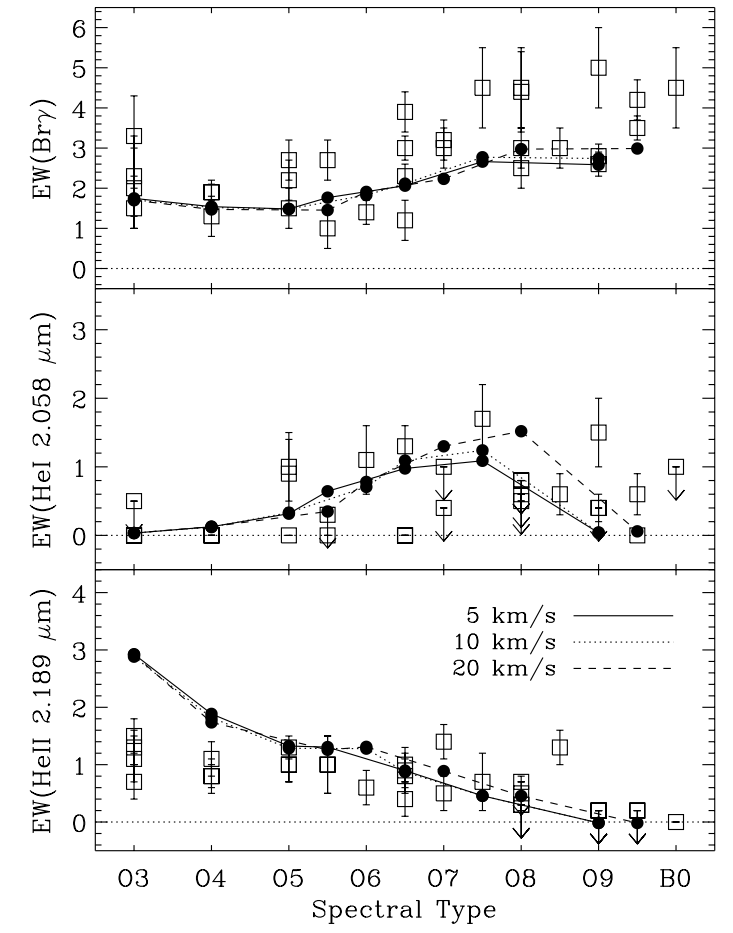

Fig. 8. Comparison between observed equivalent widths of $\mathrm{Br} \gamma$, He I $\lambda 2.058$ and He II $\lambda 2.189$ in main sequence stars (open squares, Hanson et al. 1996) and those measured from our models with $v_{\text {turb }}=5$ (solid line), 10 (dotted line) and $20 \mathrm{~km} \mathrm{~s}^{-1}$ (dashed line).

metallicity from 2 to $1 / 10 Z_{\odot}$ produces a decrease in the equivalent width of the He II line from 3.3 to $1.8 \AA$. Br $\gamma$ shows only variations at the $30 \%$ level and the He I line is practically absent at all metallicities (equivalent width $<0.1 \AA$ ). In the case of model \#5 with $v_{\text {turb }}=10 \mathrm{~km} \mathrm{~s}^{-1}$ (an O6 star), the equivalent width of the He I line remains practically unchanged; the $\mathrm{Br} \gamma$ and He II line show variations of the order of 20-30\%.

Summing up, variations in microturbulent velocity have no significant impact on the equivalent width of the $\mathrm{Br} \gamma$, $\mathrm{He}$ I and He II lines, while variations in metal content have an appreciable influence on the equivalent width of the He II line in the case of very hot stars.

\section{The ionizing structure of single star $\mathrm{H} \|$ regions}

Observations of $\mathrm{H}$ II regions combined with detailed photoionization models can be used to test the EUV spectrum of O stars. In this respect, the Infrared Space Observatory (ISO, Kessler et al. 1996) provided an unique opportunity by measuring the infrared (2.3-196 $\mu \mathrm{m})$ spectra of a large sample of H II regions (Giveon et al. 2002; Peeters et al. 2002; Vermeij et al. 2002), giving access to four elements (N, Ne, S and Ar) in two different ionization stages. Ratios of fine-structure lines such as [N III]/[N II] 57/122 $\mu \mathrm{m}$, [Ne III]/[Ne II] 15.5/12.8 $\mu \mathrm{m}$, [S IV]/[S III] 10.5/18.7 $\mu \mathrm{m}$ and [Ar III]/[Ar II] 9.0/7.0 $\mu \mathrm{m}$ probe the ionizing stellar spectrum between $27.6 \mathrm{eV}(450 \AA)$ and $41 \mathrm{eV}(303 \AA)$ and can be used as indicators of the realism of stellar models at these energies.

Basically, the ratio of two successive stages of ionization $X^{+\mathrm{i}}$ and $X^{+\mathrm{i}+1}$ of a given element $X$ indicates the state 


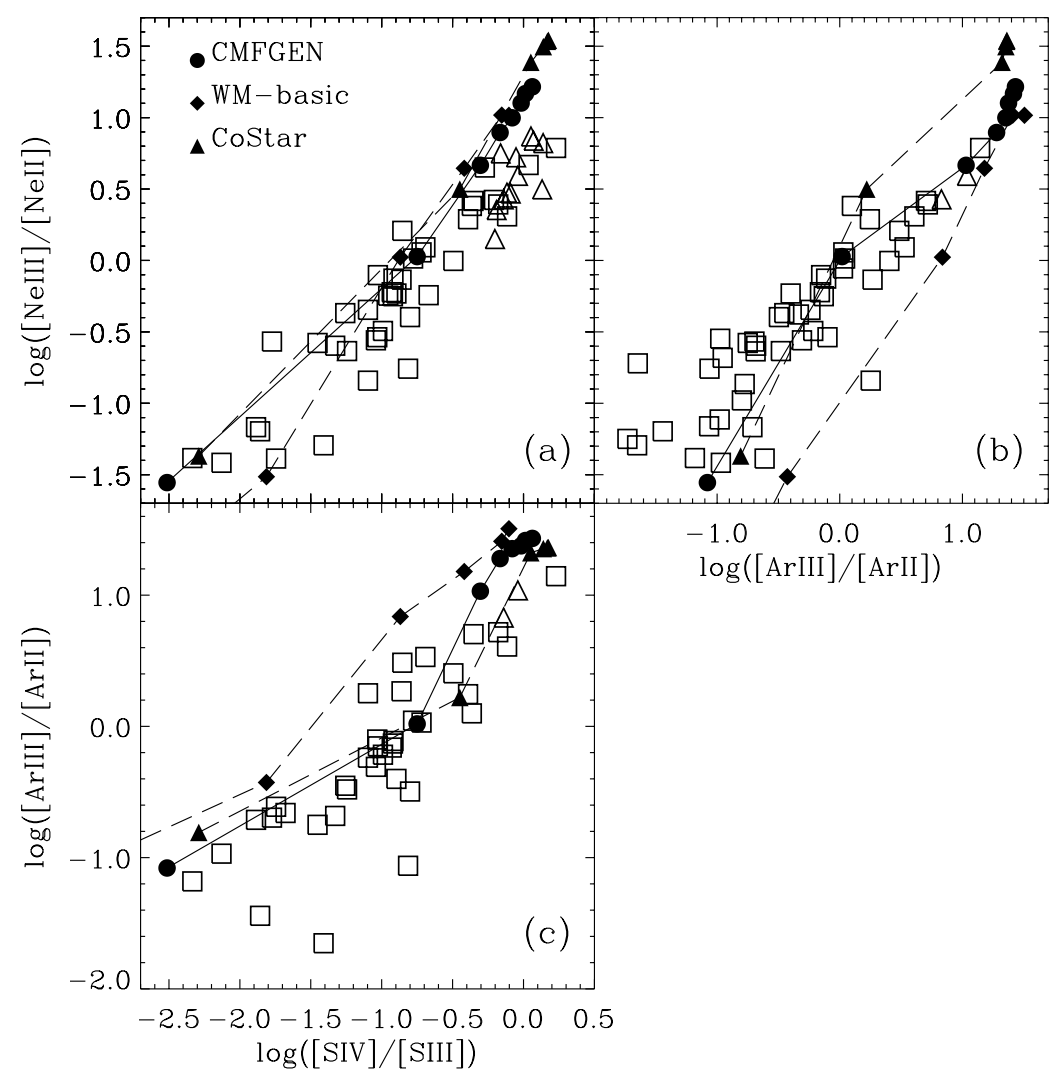

Fig. 9. Comparison of the infrared fine-structure line ratios [Ar III]/[Ar II] 9.0/7.0, [S IV]/[S III] 10.5/18.7 and [Ne III]/[Ne II] 15.5/12.8 $\mu \mathrm{m}$ observed in Galactic (open squares) and Magellanic Cloud (open triangles) H II regions with predictions from photoionization models at solar metallicity based on three different SEDS: CMFGEN (solid circles), WM-BASIC (solid diamonds) and CoSTAR (solid triangles). A unique ionization parameter, $U$, is adopted for all the models. We stress that unless $U$ is constrained, these line ratios cannot be used to derive an absolute value for $T_{\text {eff }}$.

of ionization of the nebula, which depends principally on the shape of the SED, more specifically on the number of photons able to ionize $X^{+\mathrm{i}}$ compared to that of Lyman continuum photons (Vílchez \& Pagel 1988), and on the ionization parameter $U$. In the case of an ionized sphere of constant gas density, $n$, and filling factor, $\epsilon, U$ is defined by $U=Q_{0} /\left(4 \pi R_{\mathrm{s}}^{2} n c\right) \propto$ $\left(Q_{0} n \epsilon^{2}\right)^{1 / 3}$ (see e.g. Stasińska \& Schaerer 1997), where $R_{\mathrm{s}}$ is the radius of the Strömgren sphere and $c$ is the speed of light. Any combination of $Q_{0}, n$ and $\epsilon$ that keeps the product $Q_{0} n \epsilon^{2}$ constant results in a similar ionization structure.

In order to investigate the dependence of the finestructure line ratios on the SED, nebular models have been computed with the photoionization code CLOUDY version 96.00-beta $4^{1}$ using MICE, the IDL interface for CLOUDY created by Spoon ${ }^{2}$. We computed nebular models for static, spherically symmetric and homogeneous gas distributions with one ionizing star in the center. An inner cavity with a radius equal to $10^{17} \mathrm{~cm}$ is set, while the outer radius of the $\mathrm{HII}$ region is defined by the position where the electron temperature reaches $1000 \mathrm{~K}$. The grid of stellar models based on the CMFGEN code and described in Sect. 2 is taken to describe the incident SED. We take models with $v_{\text {turb }}=10 \mathrm{~km} \mathrm{~s}^{-1}$. The stellar spectra were rebinned to 2000 points, which is the limit

\footnotetext{
${ }^{1}$ See http://thunder.pa.uky.edu/cloudy/

2 See http://www.astro.rug.nl/ spoon/mice.html
}

of points for the input SED accepted by CLOUDY. The nebular metallicity was set equal to that of the stellar model used to calculate the SED. Finally, with the aim to explore the dependence of the fine-structure line ratios on the SED alone, we decided to fix the ionization parameter $U$, so that the variations of the line ratios are only due to variations of the SED appearance. Hence, we fix $Q_{0}$ to the typical value of $10^{48}$ photons s ${ }^{-1}$, the density to $n=10^{3} \mathrm{~cm}^{-3}$, and the filling factor to $\epsilon=1$. We stress here that unless $U$ is constrained, these line ratios cannot be used to derive an absolute value for $T_{\text {eff }}$ (Stasińska $\&$ Schaerer 1997).

We computed several sets of nebular models. First, a set at solar metallicity where the dependence on effective temperature is analyzed, and second, a set where the effective temperature is fixed and the metal content is modified. This last grid allows us to investigate the dependence of the fine-structure line ratios on metallicity.

Figure 9 presents the correlations observed between the fine-structure line ratios [Ar III]/[Ar II] 9.0/7.0 $\mu \mathrm{m}$, [S IV]/[S III] 10.5/18.7 $\mu \mathrm{m}$ and [Ne III]/[Ne II] 15.5/12.8 $\mu \mathrm{m}$ for the sample of Galactic (Giveon et al. 2002; Martín-Hernández et al. 2002a) and Magellanic Cloud H II regions (Vermeij et al. 2002) observed by ISO. The ionization potentials of Ar II, S III and Ne II are, respectively, $27.6 \mathrm{eV}$ (450 ̊), $34.8 \mathrm{eV}$ (357 $\AA$ ) and $41.0 \mathrm{eV}(303 \AA)$. We do not include the ratio $[\mathrm{N} \mathrm{III}] /[\mathrm{N}$ II $] 57 / 122 \mu \mathrm{m}$ in this figure; the ionization potential 


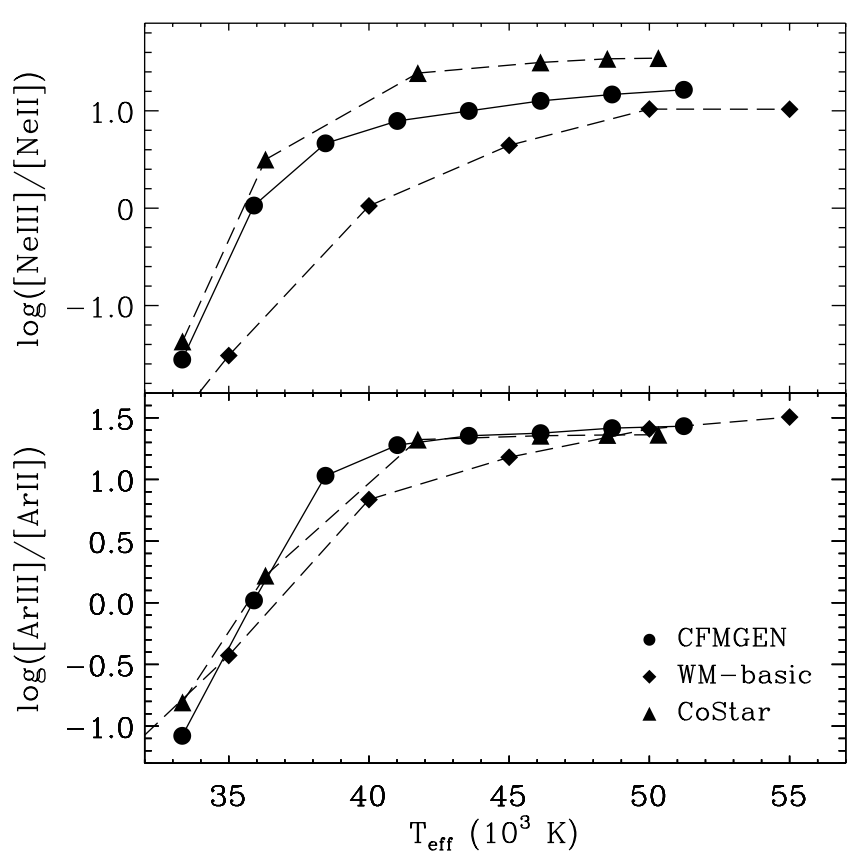

Fig. 10. Predicted [Ar III]/[Ar II $] 9.0 / 7.0 \mu \mathrm{m}$ and $[\mathrm{Ne}$ III]/[Ne II] $15.5 / 12.8 \mu \mathrm{m}$ fine-structure line ratios as a function of $T_{\text {eff }}$ for the three sets of models at solar metallicity considered in the text. A unique ionization parameter, $U$, is adopted for all the models. We stress that unless $U$ is constrained, these line ratios cannot be used to derive an absolute value for $T_{\text {eff }}$.

of $\mathrm{N}$ II $(29.6 \mathrm{eV})$ is close to that of Ar II, and hence, this ratio roughly probes the same range of energies as $[\mathrm{Ar} \mathrm{III}] /[\mathrm{Ar} I I]$. The data span a range in ionization of more than two orders of magnitude. Interestingly, the Magellanic Cloud H II regions nicely overlap with the trend observed for the Galactic objects at the high ionization end (see Martín-Hernández et al. 2002b).

The diagrams in Fig. 9 probe the shape or hardness of the stellar spectrum between 303 and $357 \AA$ (panel a), 303 and $450 \AA$ (panel b), and 357 and $450 \AA$ (panel c). In this diagram the results for the set of nebular models at solar metallicity are confronted to the observations. As a comparison, we also show the results of grids of models computed using the WM-BASIC and COSTAR stellar models. We use WM-BASIC models D-30, D-35, D-40, D-45, D-50 and D-55 (Pauldrach et al. 2001), and CoSTAR models A2, B2, C2, D2, E2 and F2 (Schaerer \& de Koter 1997). For all model sets, the predicted nebular excitation increases with increasing effective temperature of the ionizing star. However, differences among the models, and between the models and the observations, are clearly present. The largest differences are seen in panels (b) and (c), for which the range of energies tested is larger. Several conclusions can be gleaned from the inspection of Fig. 9. (1) For the same nebular conditions, i.e. same ionization parameter $U$, the nebular models based on the COSTAR SEDs with $T_{\text {eff }}>36000 \mathrm{~K}$ largely overpredict $[\mathrm{Ne}$ III]/[Ne II] for a given [S IV]/[S III] or [Ar III]/[Ar II] when compared to the observations and the other SEDs. This can be understood in terms of an excess of photons below $303 \AA$. This effect is evident in Fig. 1, which clearly shows that the COSTAR stellar spectra are much harder at these energies than the other two atmosphere

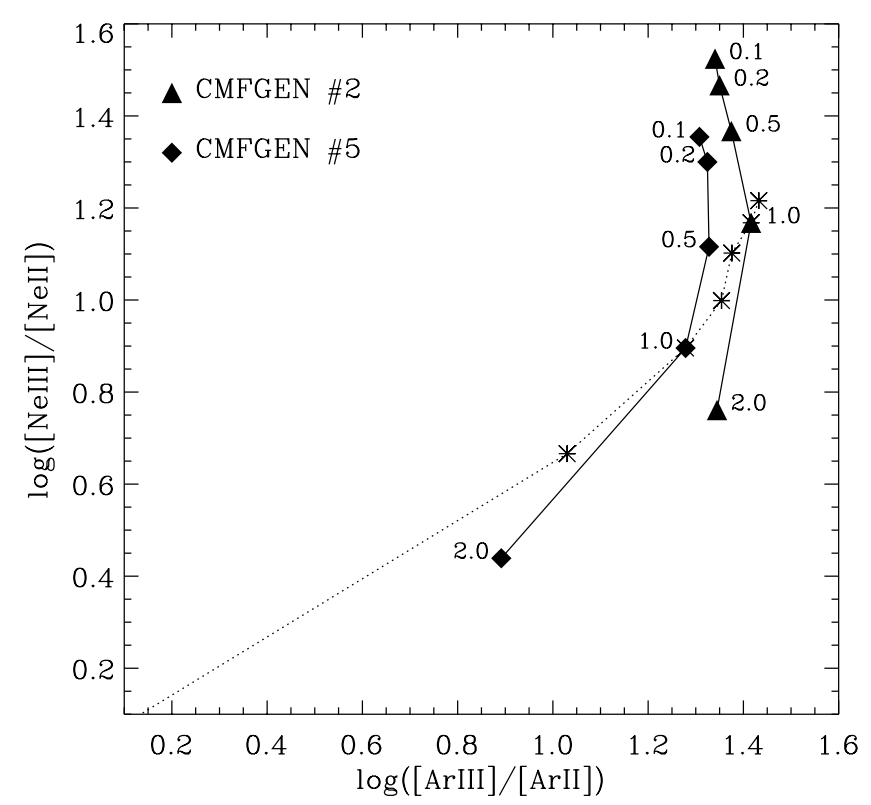

Fig. 11. Variation of the nebular fine-structure line strength ratios [Ar III]/[Ar II] 9.0/7.0 and [Ne III]/[Ne II] 15.5/12.8 $\mu \mathrm{m}$ as a consequence of modifying the stellar and nebular metallicity from 2 to $1 / 10 Z_{\odot}$. The adopted SEDs are models \#2 (solid triangles) and \#5 (solid diamonds). The dotted line connects the models at solar metallicity (star symbols, cf. Fig. 9). A unique ionization parameter, $U$, is adopted for all the models. We stress that unless $U$ is constrained, these line ratios cannot be used to derive an absolute value for $T_{\text {eff }}$.

models. (2) The nebular models based on the WM-BASIC SEDs show a too soft stellar spectrum between 303 and $450 \AA$ for the models with $T_{\text {eff }}<45000 \mathrm{~K}$, which is reflected in an underprediction of the $[\mathrm{Ne}$ III $] /[\mathrm{Ne}$ II $]$ and $[\mathrm{S} \mathrm{IV}] /[\mathrm{S} \mathrm{III}]$ line ratios for a given $[\mathrm{Ar} \mathrm{III]}] /[\mathrm{Ar} \mathrm{II}]$. This is also illustrated in Fig. 10, which shows the variation of the $[\mathrm{Ne}$ III] $] /[\mathrm{Ne}$ II] and $[\mathrm{Ar}$ III $] /[\mathrm{Ar}$ II $]$ line ratios with $T_{\text {eff }}$. The $[\mathrm{Ar} \mathrm{III}] /[\mathrm{Ar} \mathrm{II}]$ line ratio, which is sensitive to the number of photons able to ionize Ar II (below $450 \AA$ ) relative to the total number of Lyman photons, is, for a given $U$, practically independent of the stellar model used in the photoionization model. However, large discrepancies appear in the case of the $[\mathrm{Ne}$ III]/[Ne II] line ratio. This is because the range of energies probed by $[\mathrm{Ne} \mathrm{III}] /[\mathrm{Ne}$ II] depends greatly on the way the photosphere and wind parameters of the stellar atmosphere are defined. Hence, the large differences between the models. In particular, the nebular models based on the WM-BASIC SEDs predict a lower [Ne III]/[Ne II] than the other sets of models for $T_{\text {eff }}<45000 \mathrm{~K}$. (3) In the case of the nebular models based on the CMFGEN SEDs, the discrepancies with the observations are smaller than for the other two model grids, thus implying that the stellar spectrum between 303 and $450 \AA$ is better described by CMFGEN.

Finally, we quantify how variations in metallicity modify the ionization structure of $\mathrm{H}$ II regions and hence, the interpretation of the above fine-structure line ratios in terms of the stellar content of the nebula. Observationally, the [Ne III]/[Ne II] line ratio observed in $\mathrm{H}$ II regions show a loose correlation with nebular metallicity (Martín-Hernández et al. $2002 b$ ). Figure 11 shows the effect of varying the metallicity for 
models \#2 and \#5 on the [Ar III]/[Ar II] and [Ne III]/[Ne II] line strength ratios. In Sect. 3.3 it was discussed that, for a given effective temperature, the stellar spectrum softens with increasing metallicity. This effect has a clear impact on the degree of ionization of the $\mathrm{H}$ II region: the fine-structure line ratios decrease with increasing metallicity. The biggest effect occurs for the $[\mathrm{Ne} \mathrm{III}] /[\mathrm{Ne}$ II] ratio, which gets reduced by about a factor of 10 when the metallicity increases from $1 / 10$ to $2 Z_{\odot}$. Hence, the effect of metallicity is such that, for instance, the ionization structure as traced by $[\mathrm{Ne} \mathrm{III}] /[\mathrm{Ne}$ II] of a half solar metallicity nebula ionized by a star with $T_{\text {eff }}=41010 \mathrm{~K}$ is, for the same ionization parameter, almost identical to that of a solar metallicity nebula with a central star of $T_{\text {eff }}=48670 \mathrm{~K}$ conform Fig. 11 (also see Morisset et al. 2003).

Infrared line ratios have been used to infer the stellar content of H II regions (e.g. Takahashi et al. 2000; Okamoto et al. 2001; Morisset et al. 2002), and to constrain the ages in starbursts (e.g. Crowther et al. 1999; Thornley et al. 2000; Spoon et al. 2000). However, the large dependence of these fine-structure line ratios on metallicity make them unsuitable for such studies, unless the metallicity is known, and moreover, its influence on the stellar energy distribution is properly accounted for.

\section{Conclusions}

We have presented a grid of eight main-sequence (dwarf) star models ranging in effective temperature from 33000 up to $51000 \mathrm{~K}$. The models were constructed using the CMFGEN code of Hillier \& Miller (1998). In order to investigate the variations of the spectral appearance on metallicity, and hence, their implications for spectral classification and the ionization structure of $\mathrm{H}$ II regions, we have calculated additional sets of models in which we varied the metal content from 2 to $1 / 10 Z_{\odot}$. The main conclusions of this study are:

- The total number of Lyman photons emitted is found to be almost independent of line blanketing effects and metallicity for a given effective temperature. This is because the flux that is blocked by the forest of metal lines at $\lambda<$ $600 \AA$ is redistributed mainly within the Lyman continuum. Though some flux is removed from the ionizing continuum, the softening of the spectrum helps to conserve the number of ionizing photons.

- We investigate the influence of metallicity, microturbulent velocity and gravity $g$ on the optical lines used to spectral type O stars: He I $\lambda 4471$ and He II $\lambda 4542$. We find that spectral type, as defined by the ratio of the equivalent widths of these lines, is not only a function of the effective temperature of the star and its luminosity class, but it depends also, albeit to a lower degree, on the microturbulent velocity of the stellar atmosphere, on metallicity and, within the luminosity class of dwarfs, on gravity. A change in $v_{\text {turb }}$ from 5 to $20 \mathrm{~km} \mathrm{~s}^{-1}$ can shift the spectral type by up to half a subclass (to a later type). A change in metallicity from $1 / 10$ to 2 times the solar metallicity can shift the spectral type up to a subclass (to an earlier type). A decrease in $\log g$ of 0.3 dex implies a shift of about one spectral type (to an earlier type).

- We confirm the decrease in $T_{\text {eff }}$ for a given spectral type due to the inclusion of line blanketing. In particular, compared to the calibration of Vacca et al. (1996), which is based on plane-parallel, pure hydrogen and helium models, we find a decrease which varies between $\sim 2500$ and $\sim 3800 \mathrm{~K}$. The decrease is the largest for stars in the spectral range of 05 to $\mathrm{O} 9$.

- The comparison with the stellar features observed in the $K$-band show that variations in the microturbulent velocity do not have a significant impact on the equivalent width of the $\mathrm{Br} \gamma, \mathrm{He} \mathrm{I}$ and $\mathrm{He}$ II lines. In the case of very hot stars variations in the metallicity have an appreciable influence on the equivalent width of the He II line. In comparing the observed C IV emission features, important in the spectral type calibration using this wavelength regime, the models show a discrepant behaviour. C IV is predicted to be in absorbtion while it is observed to be in emission.

- The spectral energy distribution between 303 and $450 \AA$ is best described by the CMFGEN SEDs.

- The spectral energy distribution below $\sim 450 \AA$ is shown to be highly dependent on metallicity. This is reflected by the behaviour of the nebular fine-structure line ratios such us [Ne III]/[Ne II] 15.5/12.8 and [Ar III]/[Ar II] 9.0/7.0 $\mu \mathrm{m}$. The dependence of these line ratios on metallicity complicates their use as diagnostic tools for the effective temperature of the ionizing stars in H II regions and the age dating of starburst regions in galaxies.

Acknowledgements. We thank the referee Rolf Kudritzki for his critical reading and constructive comments, in particular with respect to the optical spectral classification, which have improved this paper substantially. We are also grateful to John Hillier for his assistance in setting up the model grid. M.R.M. acknowledges financial support from the NWO Council for Physical Sciences. Model calculations were carried out using the Beowulf cluster of the University of Amsterdam. MICE is supported at MPE by DLR (DARA) under grants 50 QI 86108 and 50 QI 94023.

\section{References}

Audouze, J. 1987, in Observational Cosmology, IAU Symp., 124, 89 Bik, A., Lenorzer, A., Kaper, L., et al. 2003, A\&A, 404, 249

Bresolin, F., \& Kennicutt, R. C. 2002, ApJ, 572, 838

Bresolin, F., Kennicutt, R. C., \& Garnett, D. R. 1999, ApJ, 510, 104

Conti, P. S. 1973, ApJ, 179, 161

Conti, P. S., \& Alschuler, W. R. 1971, ApJ, 170, 325

Conti, P. S., \& Frost, S. A. 1977, ApJ, 212, 728

Cox, A. N. 2000, Allen's astrophysical quantities, 4th ed. (New York: AIP Press; Springer), ed. A. N. Cox., ISBN: 0387987460

Crowther, P. A., Beck, S. C., Willis, A. J., et al. 1999, MNRAS, 304, 654

Crowther, P. A., Hillier, D. J., Fullerton, A. W., \& De Marco, O. 2001, Am. Astron. Soc. Meet., 199

de Koter, A., Heap, S. R., \& Hubeny, I. 1997, ApJ, 477, 792

de Koter, A., Heap, S. R., \& Hubeny, I. 1998, ApJ, 509, 879

de Koter, A., Schmutz, W., \& Lamers, H. J. G. L. M. 1993, A\&A, 277, 561

Feldmeier, A., Pauldrach, A., \& Puls, J. 1998, in Properties of Hot Luminous Stars, ASP Conf. Ser., 131, 278 
Gabler, R., Gabler, A., Kudritzki, R. P., Puls, J., \& Pauldrach, A. 1989, A\&A, 226, 162

Giveon, U., Sternberg, A., Lutz, D., Feuchtgruber, H., \& Pauldrach, A. W. A. 2002, ApJ, 566, 880

Groenewegen, M. A. T., \& Lamers, H. J. G. L. M. 1989, A\&AS, 79, 359

Hanson, M. M., Conti, P. S., \& Rieke, M. J. 1996, ApJS, 107, 281

Hanson, M. M., Rieke, G. H., \& Luhman, K. L. 1998, AJ, 116, 1915

Henry, R. B. C., \& Worthey, G. 1999, PASP, 111, 919

Herrero, A. 2003, in A Massive Star Odyssey: From Main Sequence to Supernova, IAU Symp., 212, 3

Herrero, A., Kudritzki, R. P., Vilchez, J. M., et al. 1992, A\&A, 261, 209

Herrero, A., Puls, J., \& Najarro, F. 2002, A\&A, 396, 949

Hillier, D. J., \& Miller, D. L. 1998, ApJ, 496, 407

Kaper, L., Bik, A., Hanson, M., \& Comerón, F. 2002, in Hot Star Workshop III: The Earliest Stages of Massive Star Birth, ed. P. A. Crowther, ASP Conf. Ser., 267, 95

Kessler, M. F., Steinz, J. A., Anderegg, M. E., et al. 1996, A\&A, 315, L27

Kholopov, P. N., Samus, N. N., Frolov, M. S., et al. 1998, in Combined General Catalogue of Variable Stars, 4.1 Ed (II/214A), 0

Kudritzki, R., \& Puls, J. 2000, ARA\&A, 38, 613

Kudritzki, R. P. 2002, ApJ, 577, 389

Kudritzki, R. P., Simon, K. P., \& Hamann, W.-R. 1983, A\&A, 118, 245

Lamers, H. J. G. L. M., Snow, T. P., \& Lindholm, D. M. 1995, ApJ, 455,269

Lanz, T., de Koter, A., Hubeny, I., \& Heap, S. R. 1996, ApJ, 465, 359

Lanz, T., \& Hubeny, I. 2003, ApJS, 146, 417

Leitherer, C., Robert, C., \& Drissen, L. 1992, ApJ, 401, 596

Lenorzer, A., Vandenbussche, B., Morris, P., et al. 2002, A\&A, 384, 473

MacFarlane, J. J., Cohen, D. H., \& Wang, P. 1994, ApJ, 437, 351

Martín-Hernández, N. L., Bik, A., Kaper, L., Tielens, A. G. G. M., \& Hanson, M. M. 2003, A\&A, 405, 175

Martín-Hernández, N. L., Peeters, E., Morisset, C., et al. 2002a, A\&A, 381,606

Martín-Hernández, N. L., Vermeij, R., Tielens, A. G. G. M., van der Hulst, J. M., \& Peeters, E. 2002b, A\&A, 389, 286

Martins, F., Schaerer, D., \& Hiller, D. J. 2002, A\&A, 382, 999

Mathys, G. 1988, A\&AS, 76, 427

Mathys, G. 1989, A\&AS, 81, 237

McErlean, N. D., Lennon, D. J., \& Dufton, P. L. 1998, A\&A, 329, 613

Meyer, M. R., Edwards, S., Hinkle, K. H., \& Strom, S. E. 1998, ApJ, 508, 397

Morisset, C., Schaerer, D., Bouret, J. C., \& Martins, F. 2003 [arXiv: astro-ph/0310151]
Morisset, C., Schaerer, D., Martín-Hernández, N. L., et al. 2002, A\&A, 386, 558

Oey, M. S., Dopita, M. A., Shields, J. C., \& Smith, R. C. 2000, ApJS, 128,511

Okamoto, Y. K., Kataza, H., Yamashita, T., Miyata, T., \& Onaka, T. 2001, ApJ, 553, 254

Pagel, B. E. J. 1992, in The Stellar Populations of Galaxies, IAU Symp., 149, 133

Pauldrach, A. W. A., Hoffmann, T. L., \& Lennon, M. 2001, A\&A, 375, 161

Pedoussaut, A., Capdeville, A., Ginestet, N., \& Carquillat, J. M. 1996, VizieR Online Data Catalog, 4016, 0

Peeters, E., Martín-Hernández, N. L., Damour, F., et al. 2002, A\&A, 381,571

Puls, J., Kudritzki, R.-P., Herrero, A., et al. 1996, A\&A, 305, 171

Puls, J., Repolust, T., Hoffmann, T. L., Jokuthy, A., \& Venero, R. O. J. 2003, in A Massive Star Odyssey: From Main Sequence to Supernova, IAU Symp., 212, 61

Schaerer, D. 2000, in Stars, Gas and Dust in Galaxies: Exploring the Links, ASP Conf. Ser., 221, 99

Schaerer, D., \& de Koter, A. 1997, A\&A, 322, 598

Smith, K. C., \& Howarth, I. D. 1998, MNRAS, 299, 1146

Smith, L. J., Norris, R. P. F., \& Crowther, P. A. 2002, MNRAS, 337, 1309

Spoon, H. W. W., Koornneef, J., Moorwood, A. F. M., Lutz, D., \& Tielens, A. G. G. M. 2000, A\&A, 357, 898

Stasińska, G., \& Schaerer, D. 1997, A\&A, 322, 615

Takahashi, H., Matsuhara, H., Watarai, H., \& Matsumoto, T. 2000, ApJ, 541, 779

Thornley, M. D., Schreiber, N. M. F., Lutz, D., et al. 2000, ApJ, 539, 641

Vacca, W. D., Garmany, C. D., \& Shull, J. M. 1996, ApJ, 460, 914

Vermeij, R., Damour, F., van der Hulst, J. M., \& Baluteau, J.-. P. 2002, A\&A, 390, 649

Vílchez, J. M., \& Pagel, B. E. J. 1988, MNRAS, 231, 257

Villamariz, M. R., \& Herrero, A. 2000, A\&A, 357, 597

Vink, J. S., de Koter, A., \& Lamers, H. J. G. L. M. 2000, A\&A, 362, 295

Vink, J. S., de Koter, A., \& Lamers, H. J. G. L. M. 2001, A\&A, 369, 574

Walborn, N. R. 1971, ApJS, 23, 257

Walborn, N. R. 1972, AJ, 77, 312

Walborn, N. R. 1973, AJ, 78, 1067

Walborn, N. R. 1976, ApJ, 205, 419

Walborn, N. R. 1982, AJ, 87, 1300

Watson, A. M., Coil, A. L., Shepherd, D. S., Hofner, P., \& Churchwell, E. 1997, ApJ, 487, 818

Watson, A. M., \& Hanson, M. M. 1997, ApJ, 490, L165 